\title{
Asymmetric Synthesis of Tetrahydrofurans through Palladium(0)-Catalyzed [3 + 2]-Cycloaddition of Vinylcyclopropanes with Ketenes
}

Mukulesh Mondal, ${ }^{\mathrm{b}}$ Manashi Panda, ${ }^{\mathrm{b}}$ Vickie McKee, ${ }^{\mathrm{a}, \mathrm{c}}$ and Nessan J. Kerrigan ${ }^{\mathrm{a}, *}$

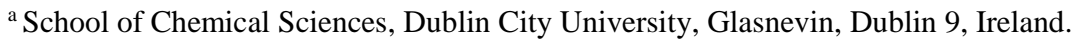

b Department of Chemistry, Oakland University, 2200 N. Squirrel Road, Rochester, MI 48309-4477, USA.

${ }^{c}$ Department of Physics, Chemistry and Pharmacy, University of Southern Denmark, Campusvej 55, 5230 Odense M, Denmark.

\section{Spectra and X-ray data}

\section{Table of Contents:}

$\mathrm{GC}$ of $( \pm)-\mathbf{1 b}$

SI-3

GC of $(S)-\mathbf{1 b}$

SI-4

$\mathrm{GC}$ of $( \pm)-1 \mathrm{c}$

$\mathrm{GC}$ of $1 \mathrm{c}$

SI-6

${ }^{1} \mathrm{H} \&{ }^{13} \mathrm{C}$ NMR of $( \pm)-3 \mathbf{a}$

${ }^{1} \mathrm{H} \&{ }^{13} \mathrm{C}$ NMR of $( \pm)-3 b$

SI-8

${ }^{1} \mathrm{H} \&{ }^{13} \mathrm{C}$ NMR of $( \pm)-3 \mathrm{c}$

${ }^{1} \mathrm{H} \&{ }^{13} \mathrm{C}$ NMR of $( \pm)-3 d$

SI-10

${ }^{1} \mathrm{H} \&{ }^{13} \mathrm{C}$ NMR of $( \pm)-3 \mathrm{e}$

${ }^{1} \mathrm{H} \&{ }^{13} \mathrm{C}$ NMR of $( \pm)-3 \mathbf{f}$

${ }^{1} \mathrm{H} \&{ }^{13} \mathrm{C}$ NMR of $( \pm)-3 \mathrm{~g}$

${ }^{1} \mathrm{H} \&{ }^{13} \mathrm{C}$ NMR of $( \pm)-3 \mathrm{~h}$

SI-14

${ }^{1} \mathrm{H} \&{ }^{13} \mathrm{C}$ NMR of ( \pm )-3i

${ }^{1} \mathrm{H} \&{ }^{13} \mathrm{C}$ NMR of ( \pm )-3j

SI-16

HPLC of ( \pm )-3b

HPLC of $\mathbf{3 k}$

SI-18

${ }^{1} \mathrm{H} \&{ }^{13} \mathrm{C}$ NMR of $\mathbf{3 k}$

SI-19

HPLC of ( \pm -3e

HPLC of 31

${ }^{1} \mathrm{H} \&{ }^{13} \mathrm{C}$ NMR of 31

HPLC of ( \pm )-3f

HPLC of 3m 
${ }^{1} \mathrm{H} \&{ }^{13} \mathrm{C}$ NMR of $\mathbf{3 m}$

SI-25

HPLC of $( \pm)-3 h$

SI-26

HPLC of $\mathbf{3 n}$

SI-27

${ }^{1} \mathrm{H} \&{ }^{13} \mathrm{C}$ NMR of $\mathbf{3 n}$

SI-28

HPLC of $( \pm)-3 \mathbf{i}$

SI-29

HPLC of 30

SI-30

${ }^{1} \mathrm{H} \&{ }^{13} \mathrm{C}$ NMR of 30

SI-31

HPLC of $( \pm)-3 p$

SI-32

HPLC of $\mathbf{3 p}$

SI-33

${ }^{1} \mathrm{H} \&{ }^{13} \mathrm{C}$ NMR of $\mathbf{3 p}$

SI-34

HPLC of $( \pm)-5 \mathbf{b}$

SI-35

HPLC of (-)-5k

SI-36

${ }^{1} \mathrm{H} \&{ }^{13} \mathrm{C}$ NMR of $( \pm)-5 \mathbf{b}$

SI-37

${ }^{1} \mathrm{H} \&{ }^{13} \mathrm{C}$ NMR of (-)-5k

SI-38

${ }^{1} \mathrm{H} \&{ }^{13} \mathrm{C}$ NMR of (+)-6k

SI-39

${ }^{1} \mathrm{H} \&{ }^{13} \mathrm{C}$ NMR of $( \pm)-7 \mathbf{b}$

SI-40

$\mathrm{X}$-ray crystal structure data for $\mathbf{6 k}$ and $\mathbf{7 b}$

SI-41 

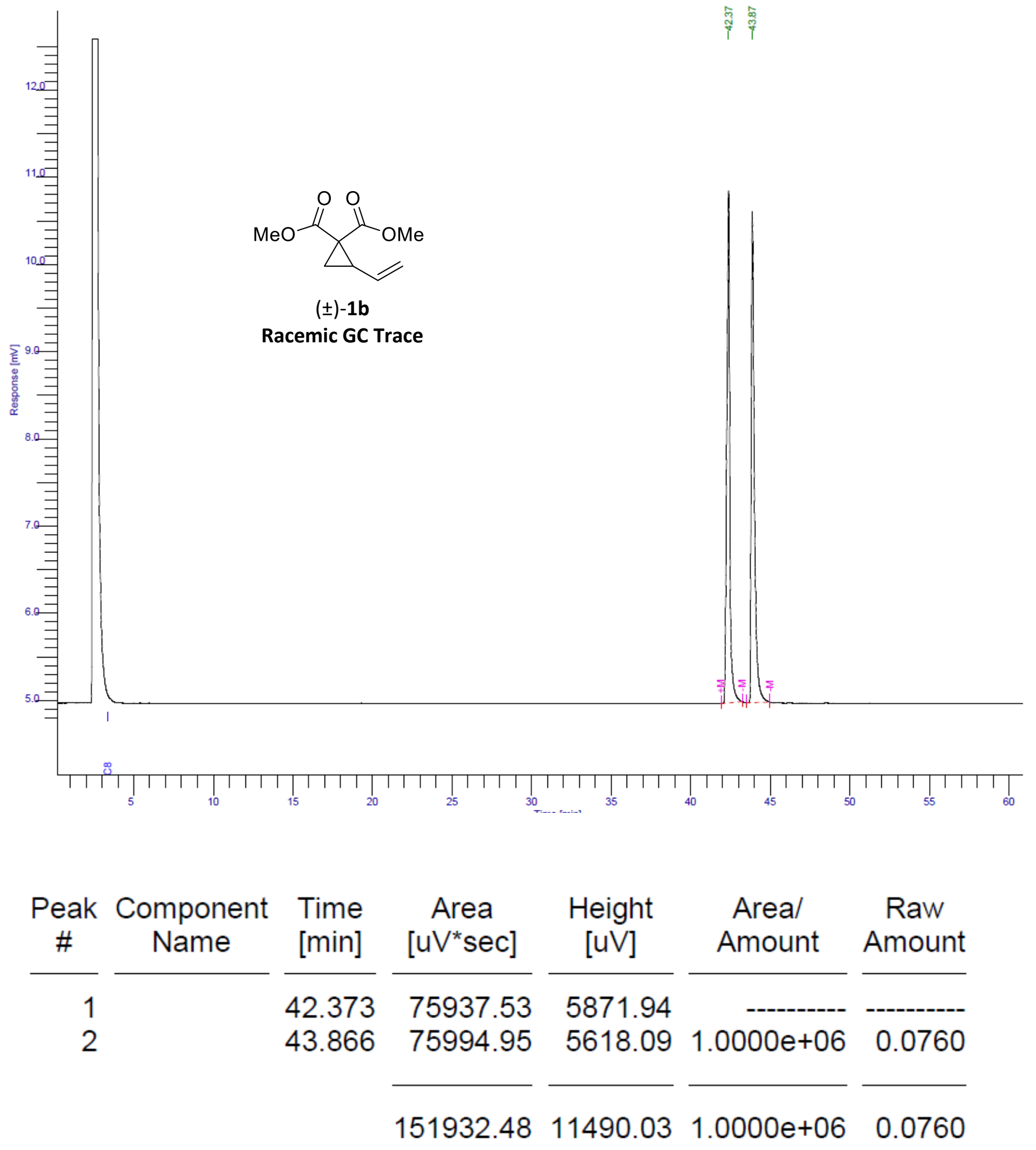

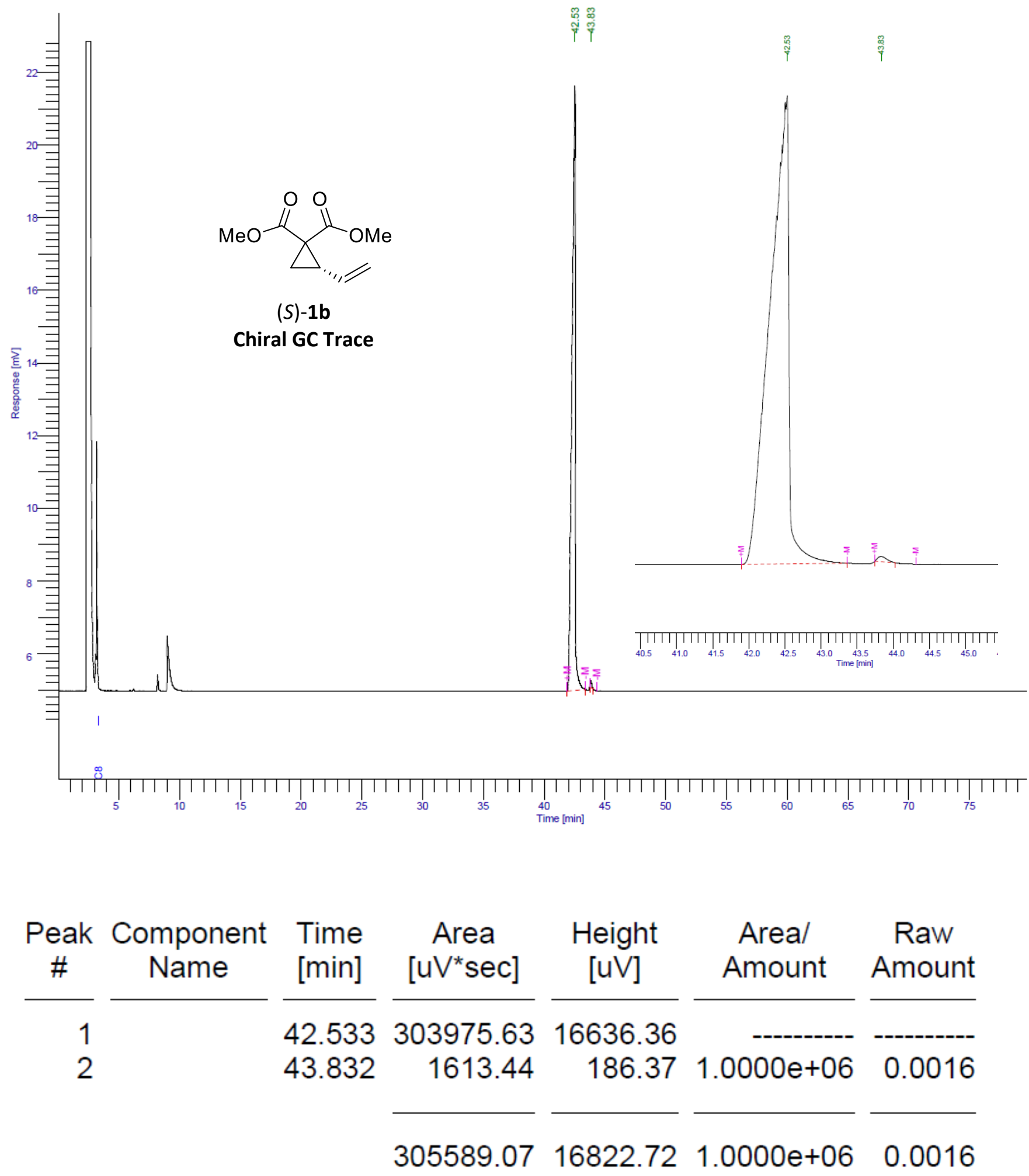

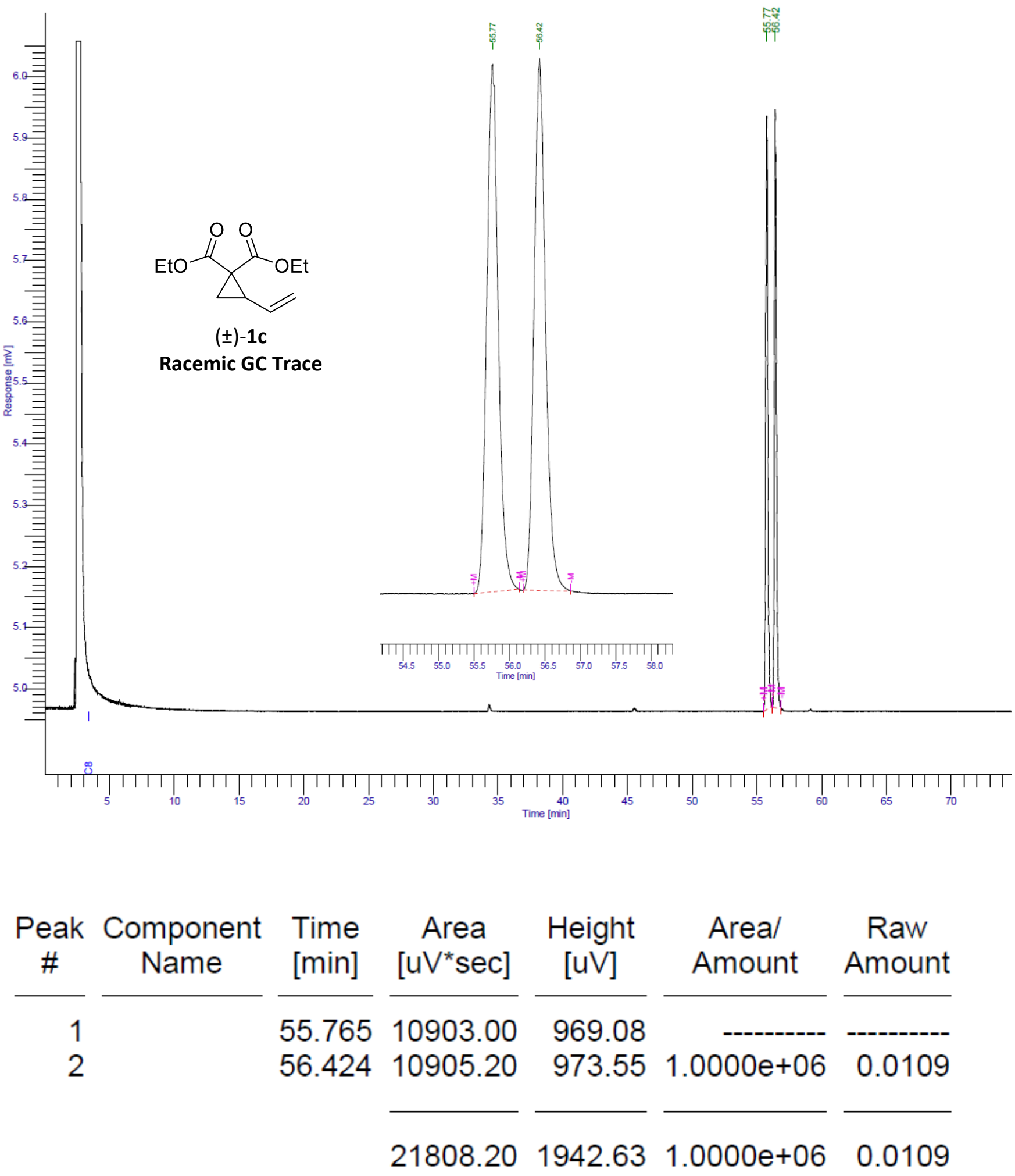

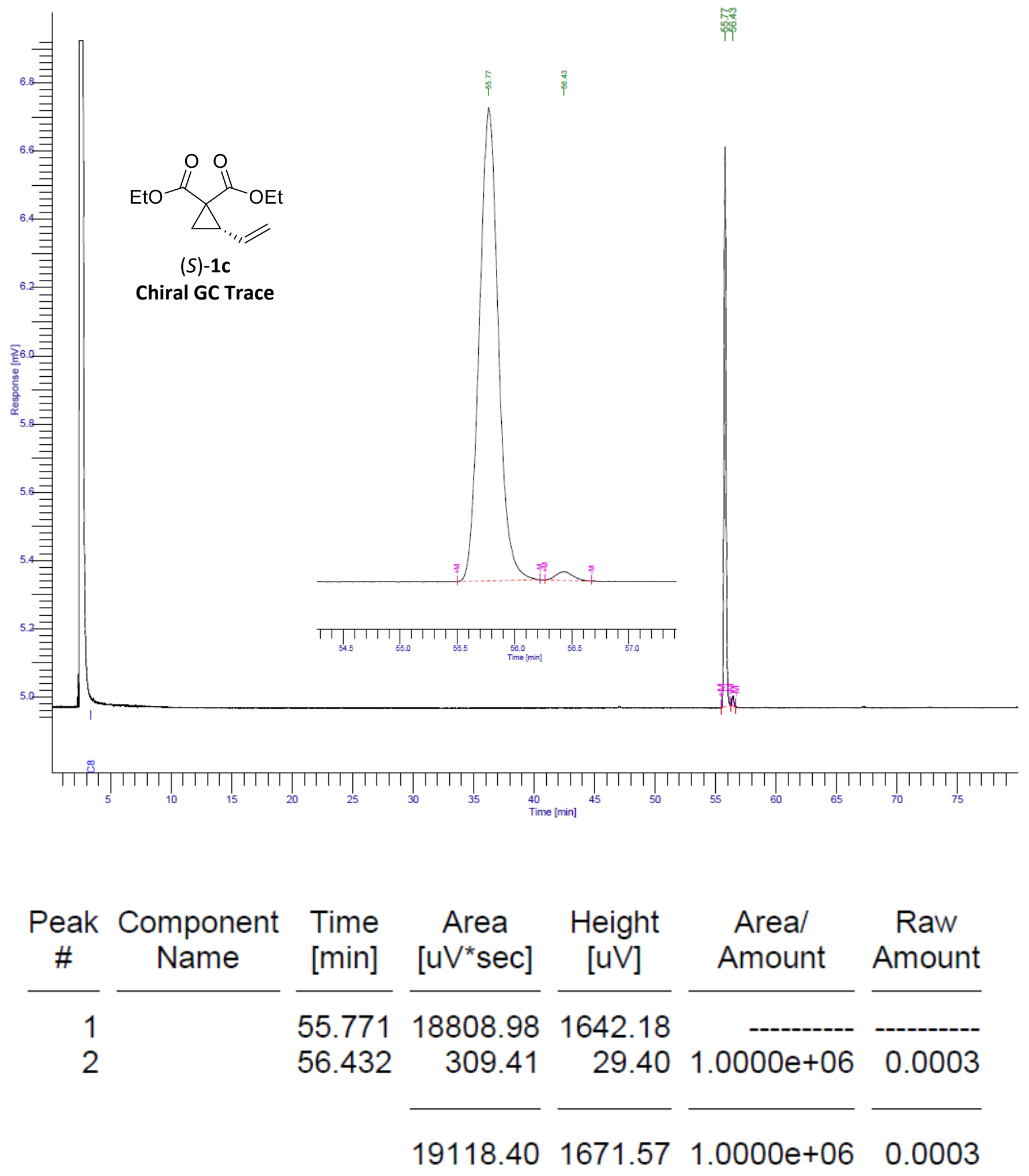


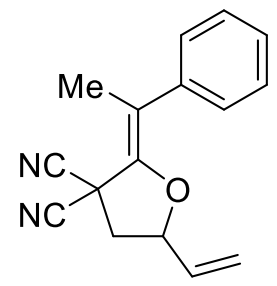

$( \pm)-3 a$

${ }^{1} \mathrm{H}$ NMR

$\mathrm{CDCl}_{3}, 400 \mathrm{MHz}$

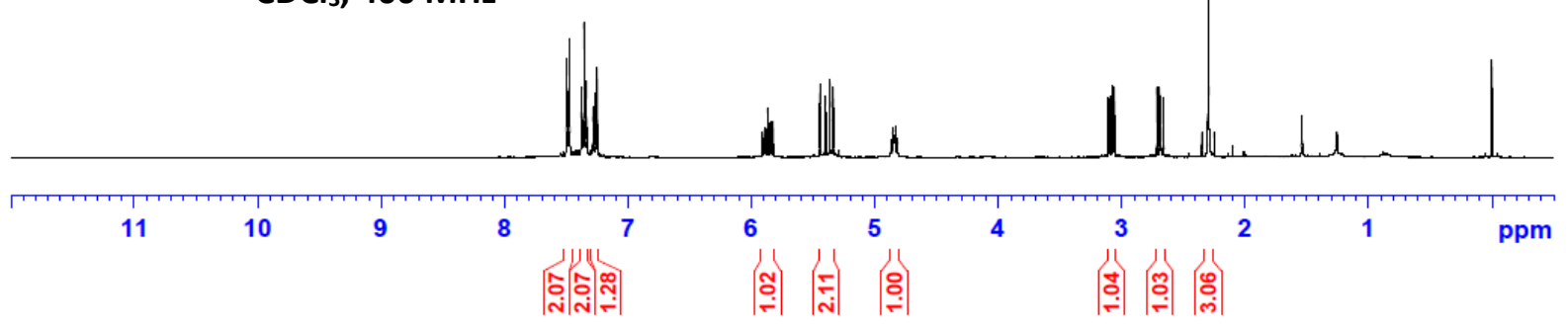

舟

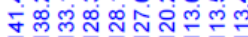

TIVIV

$\mid$

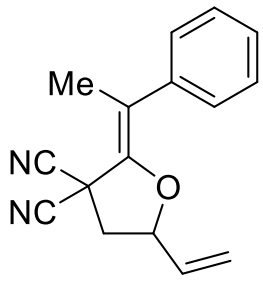

$( \pm)-3 a$

${ }^{13} \mathrm{C}$ NMR

$\mathrm{CDCl}_{3}, 100 \mathrm{MHz}$

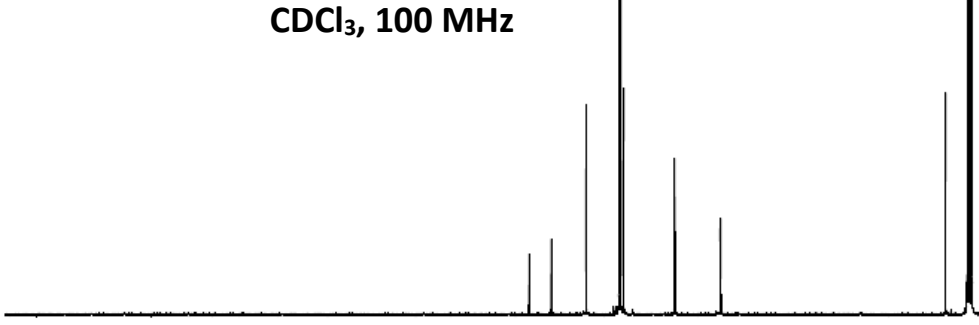

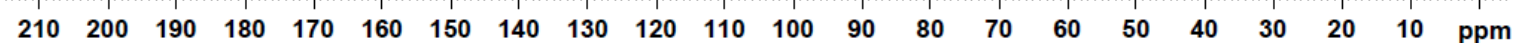



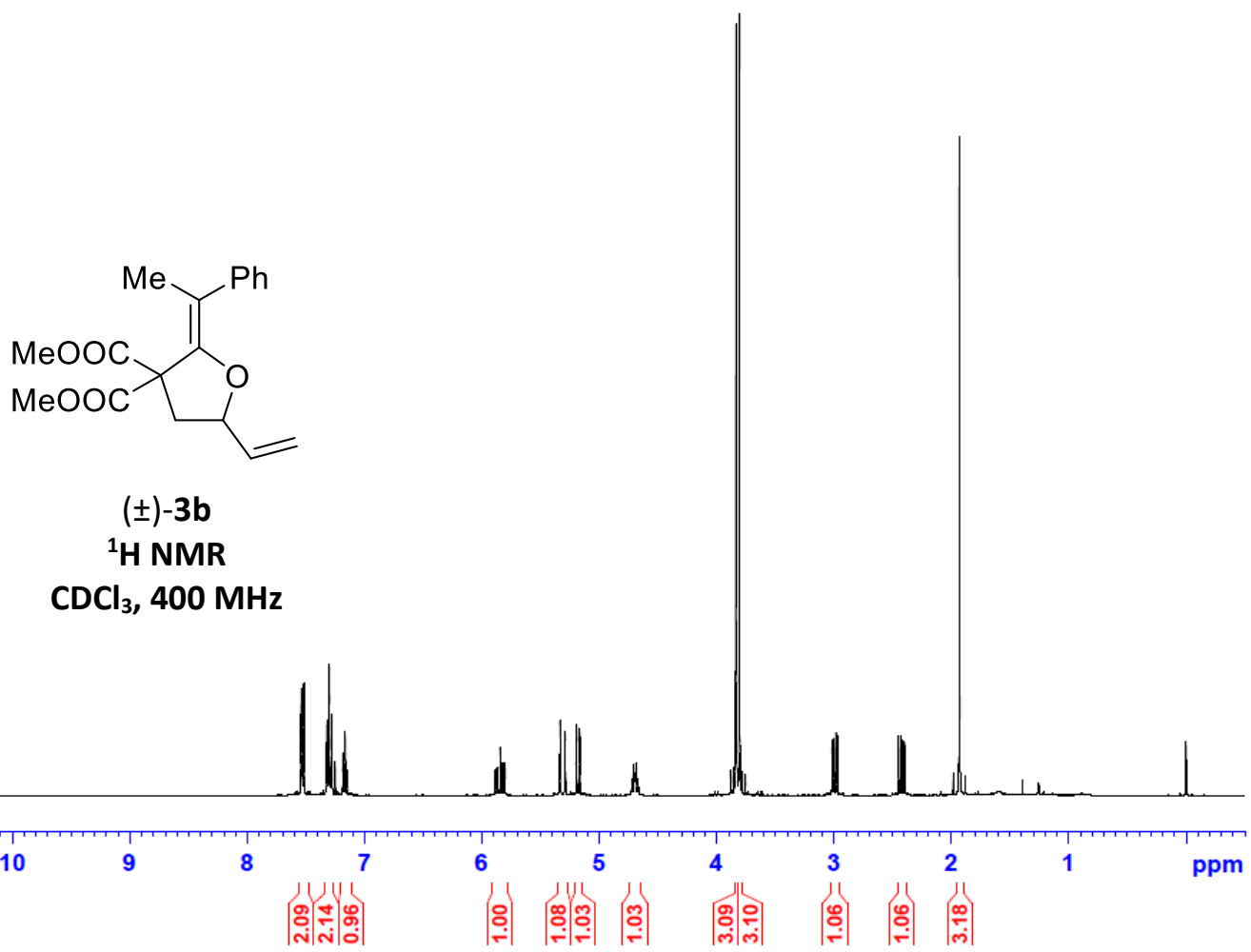

$( \pm)-3 b$

${ }^{1} \mathrm{H}$ NMR

$\mathrm{CDCl}_{3}, 400 \mathrm{MHz}$
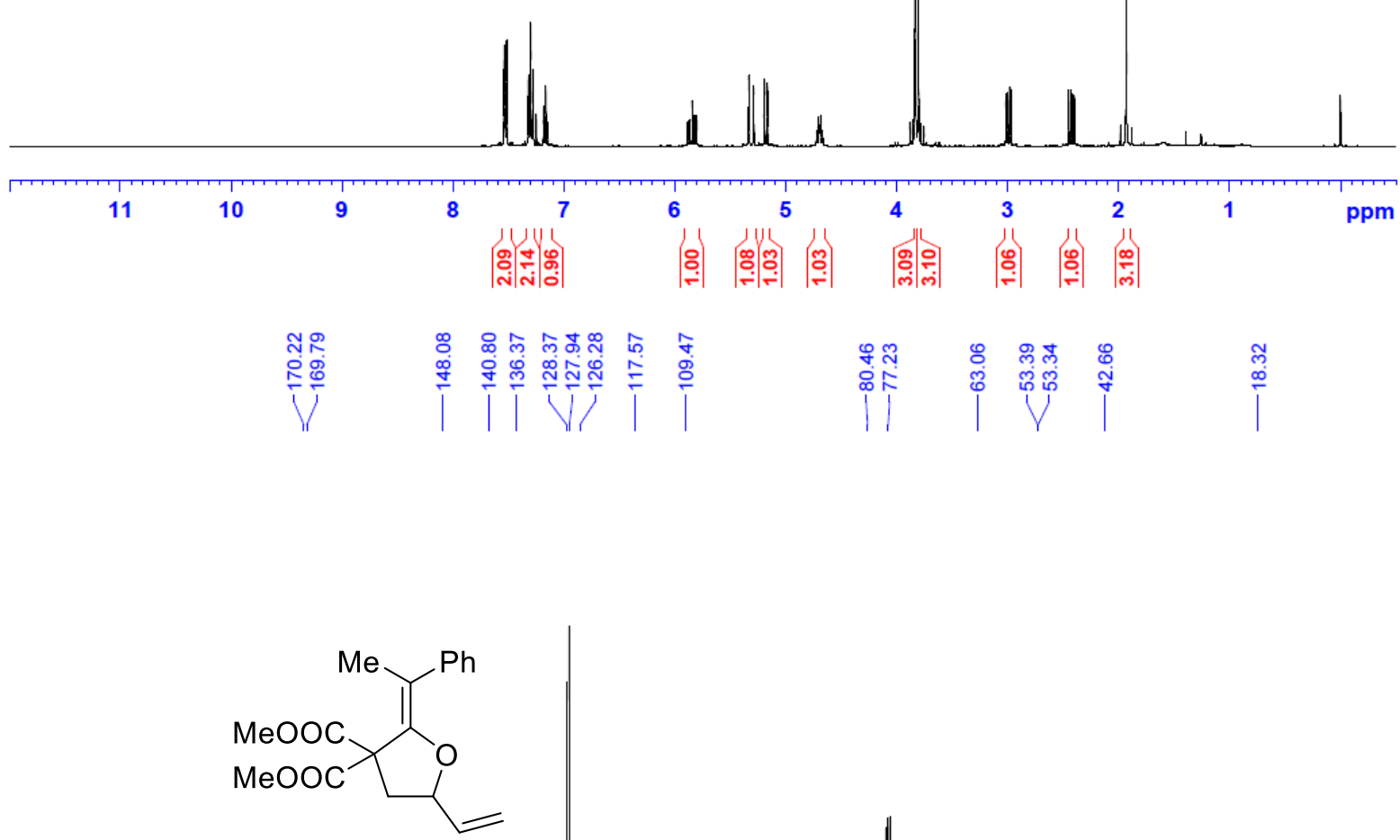

$( \pm)-3 b$

${ }^{13} \mathrm{C}$ NMR

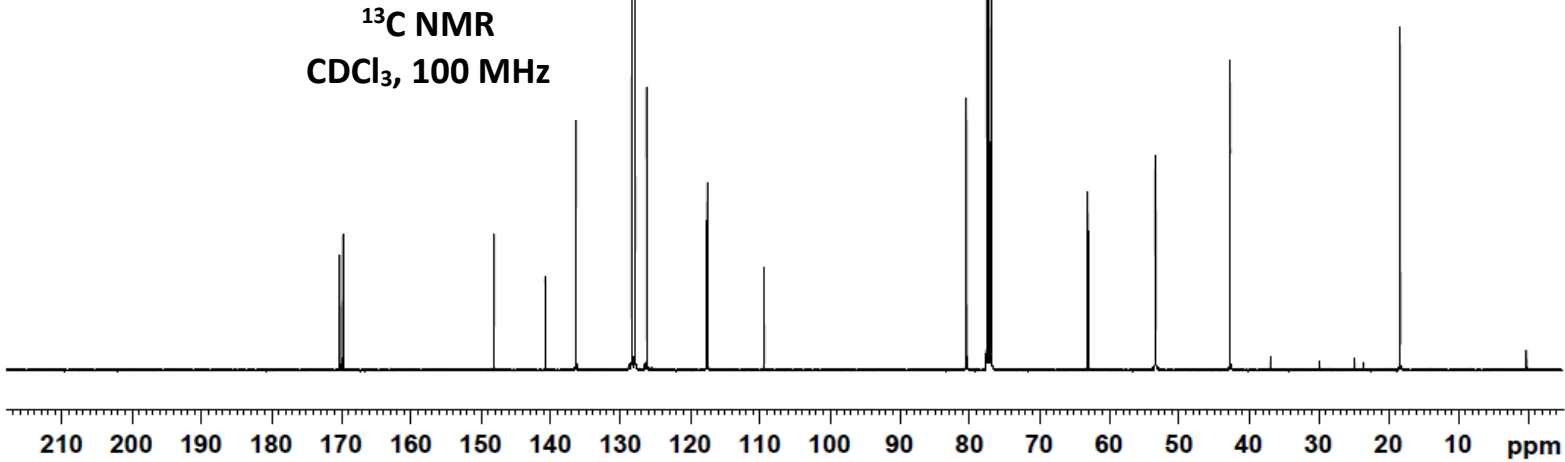




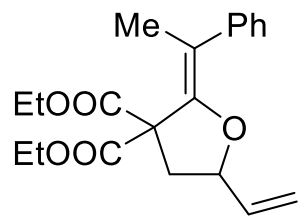

$( \pm)-3 c$

${ }^{1} \mathrm{H}$ NMR $\mathrm{CDCl}_{3}, 400 \mathrm{MHz}$

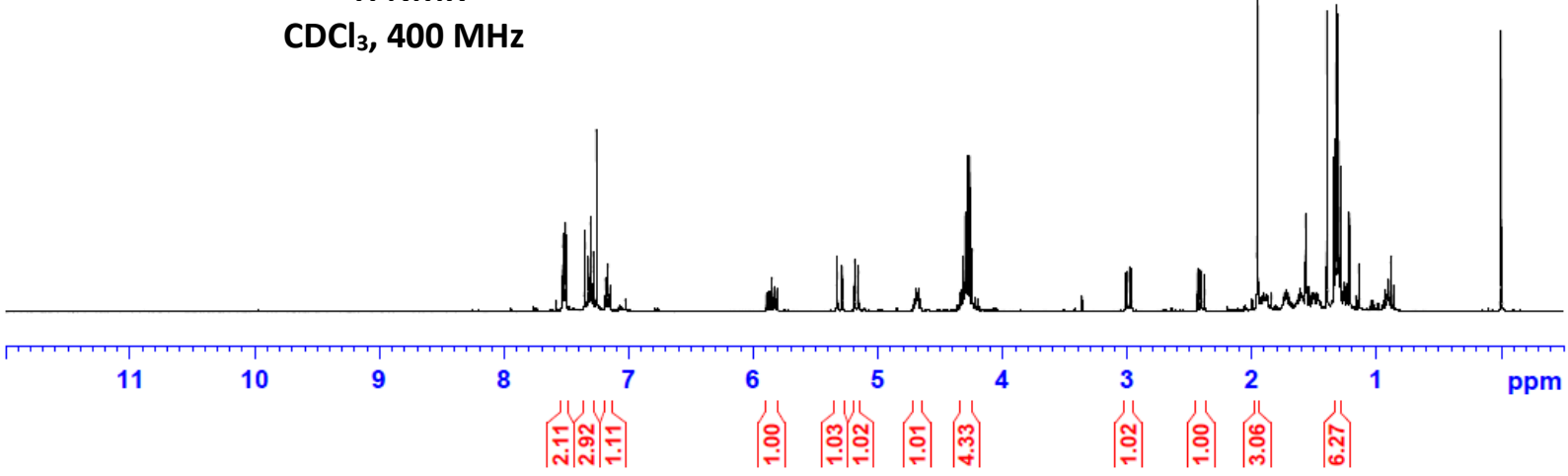

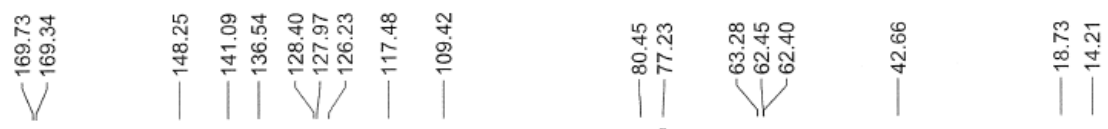

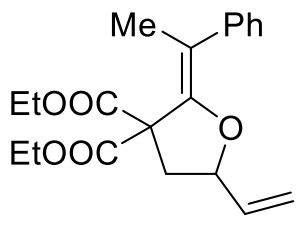

$( \pm)-3 c$

${ }^{13} \mathrm{C}$ NMR

$\mathrm{CDCl}_{3}, 100 \mathrm{MHz}$

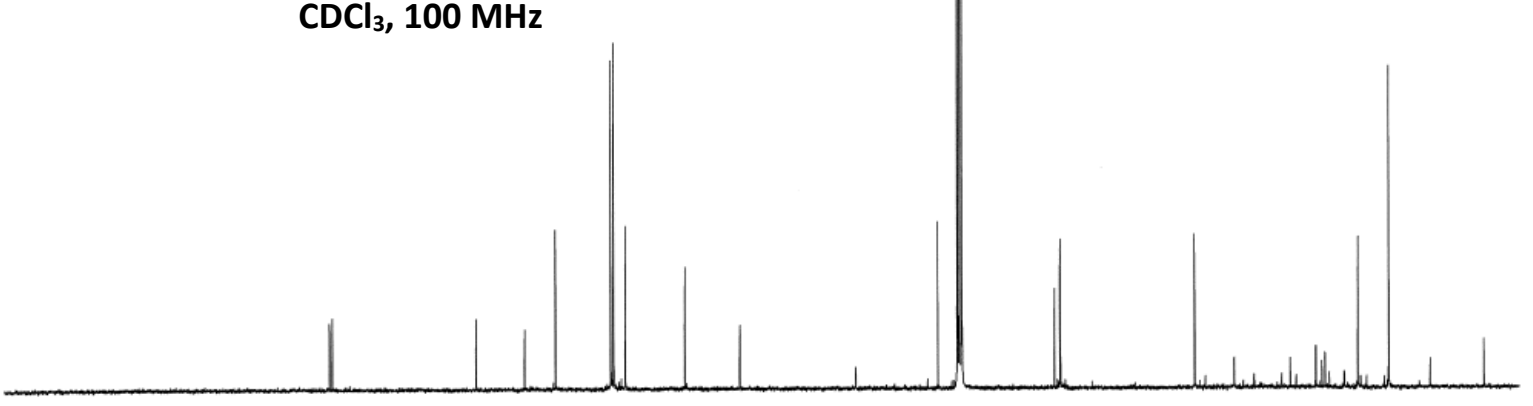

$\begin{array}{llllllllllllllllllllll}210 & 200 & 190 & 180 & 170 & 160 & 150 & 140 & 130 & 120 & 110 & 100 & 90 & 80 & 70 & 60 & 50 & 40 & 30 & 20 & 10 & \mathrm{ppm}\end{array}$ 


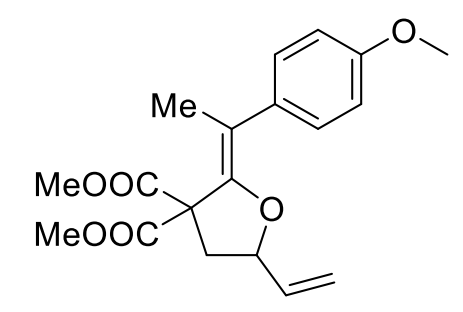

$( \pm)-3 d$

${ }^{1} \mathrm{H}$ NMR

$\mathrm{CDCl}_{3}, 400 \mathrm{MHz}$
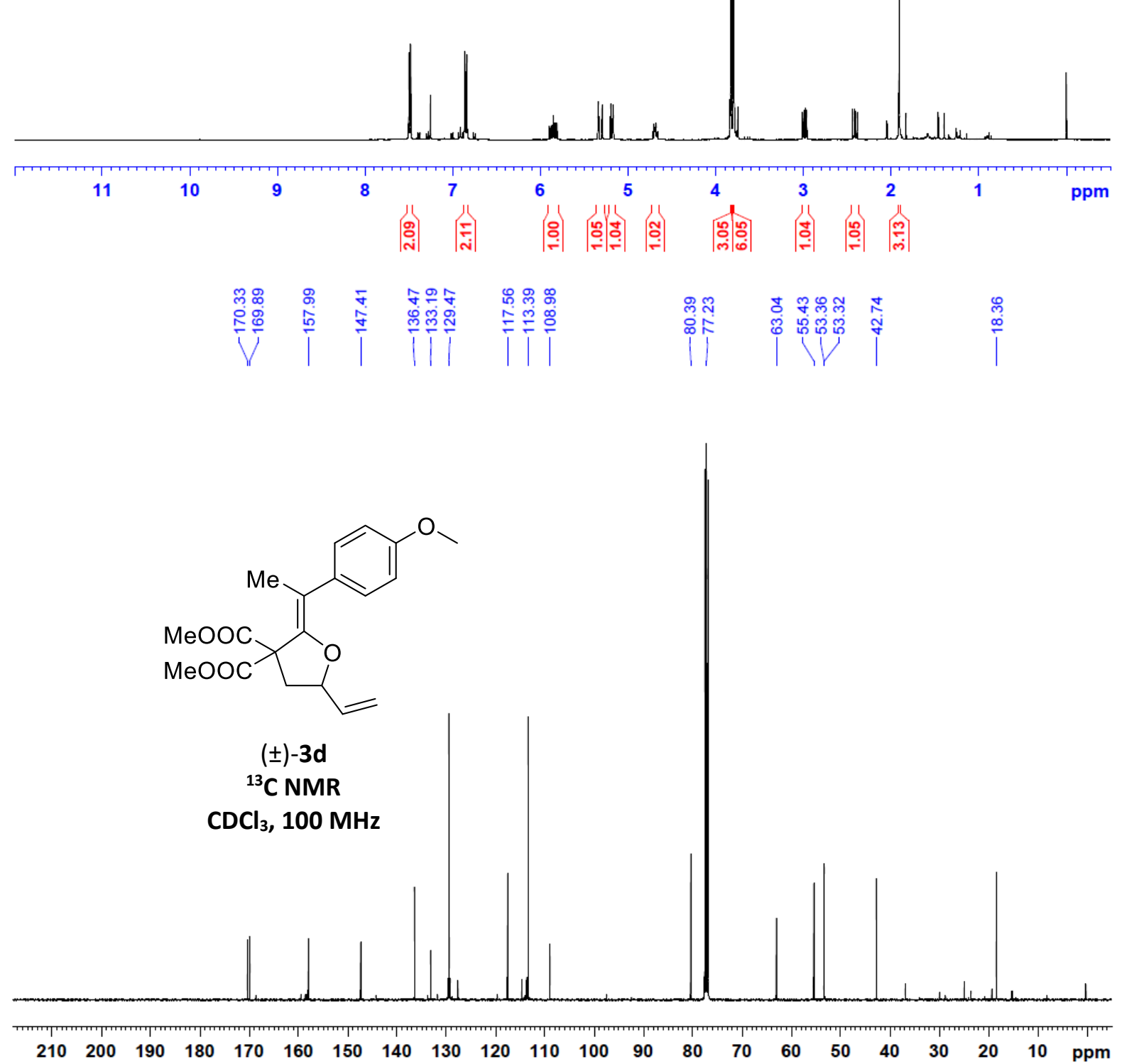


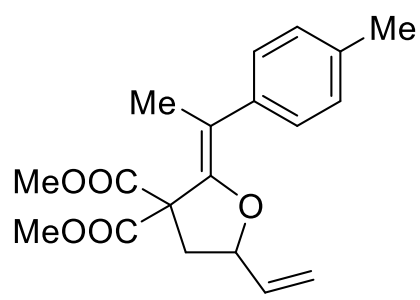

( \pm -) $3 e$

${ }^{1} \mathrm{H}$ NMR

$\mathrm{CDCl}_{3}, 400 \mathrm{MHz}$

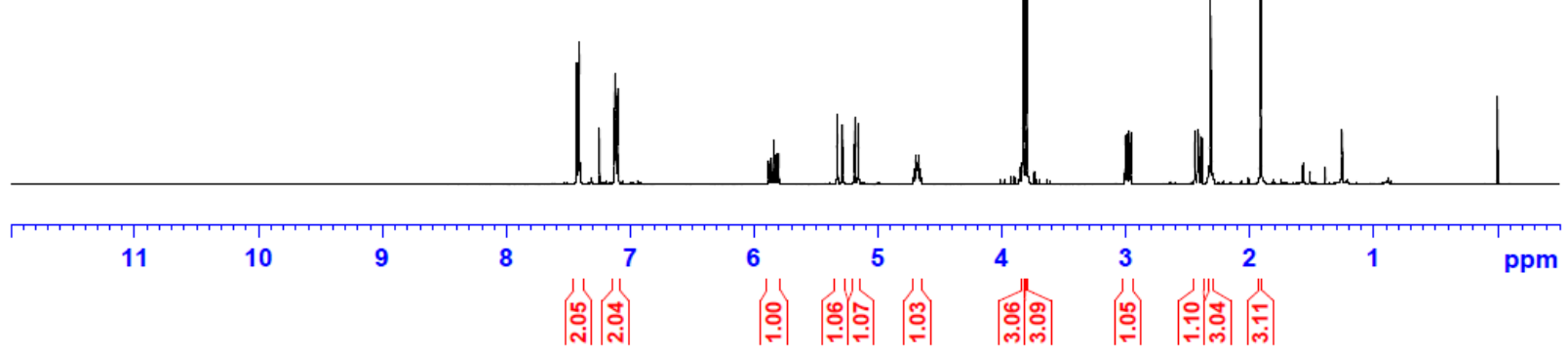

궁

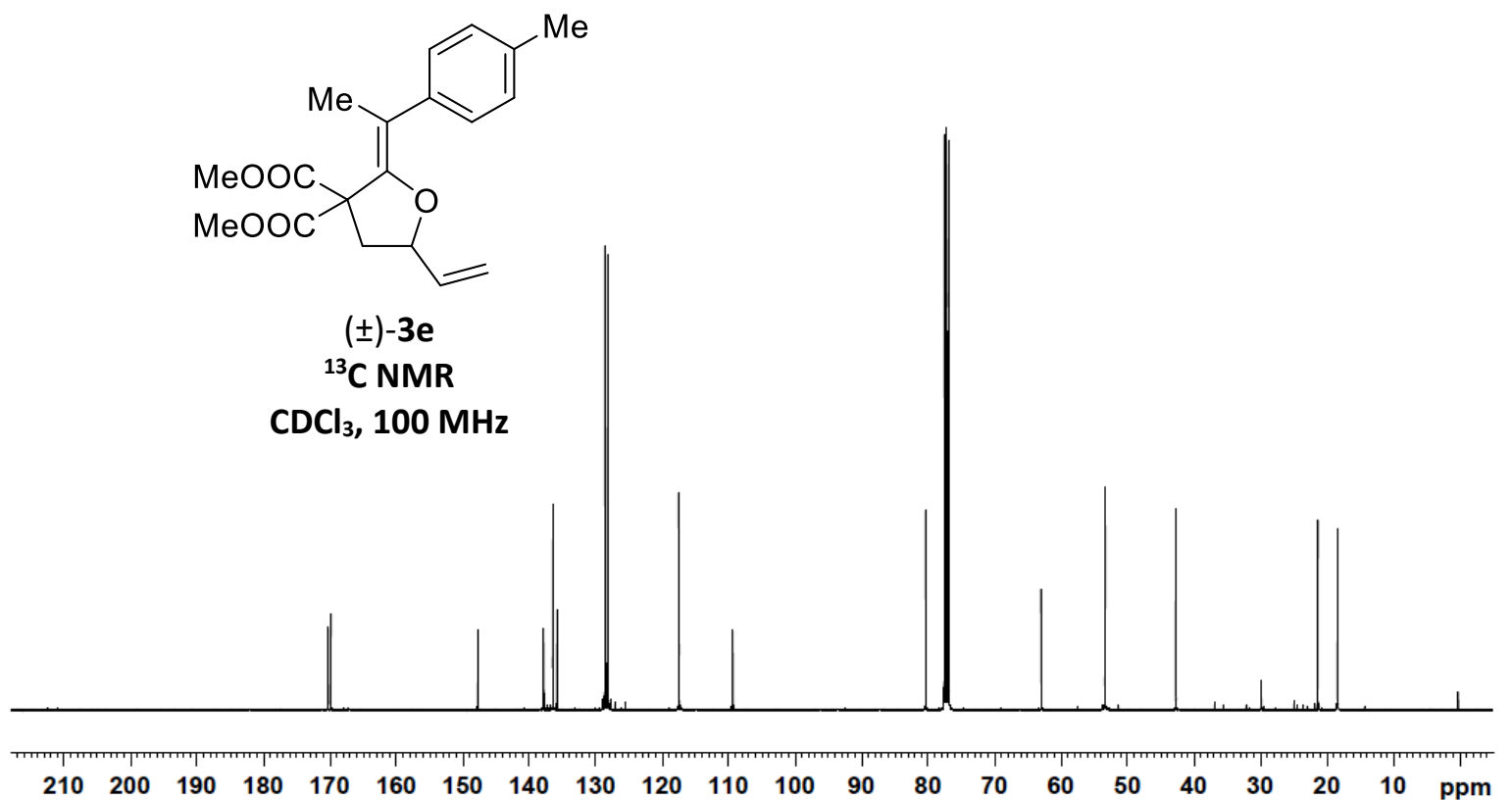




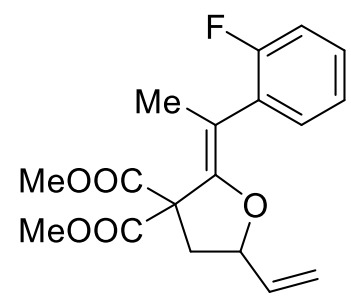

$( \pm)-3 f$

${ }^{1} \mathrm{H}$ NMR

$\mathrm{CDCl}_{3}, 400 \mathrm{MHz}$

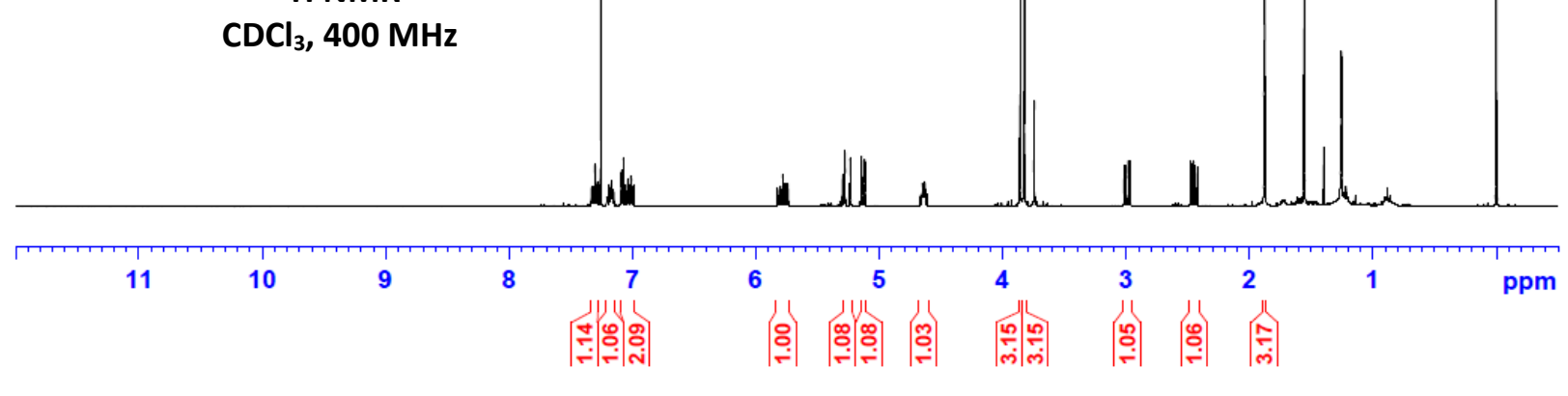

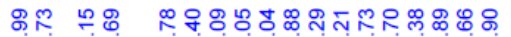

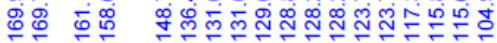

VIT

|<smiles>C=CC1CC(C(C)=O)(C(C)=O)C(=C(C)c2ccccc2F)O1</smiles>

$( \pm)-3 f$

${ }^{13} \mathrm{C}$ NMR

$\mathrm{CDCl}_{3}, 100 \mathrm{MHz}$

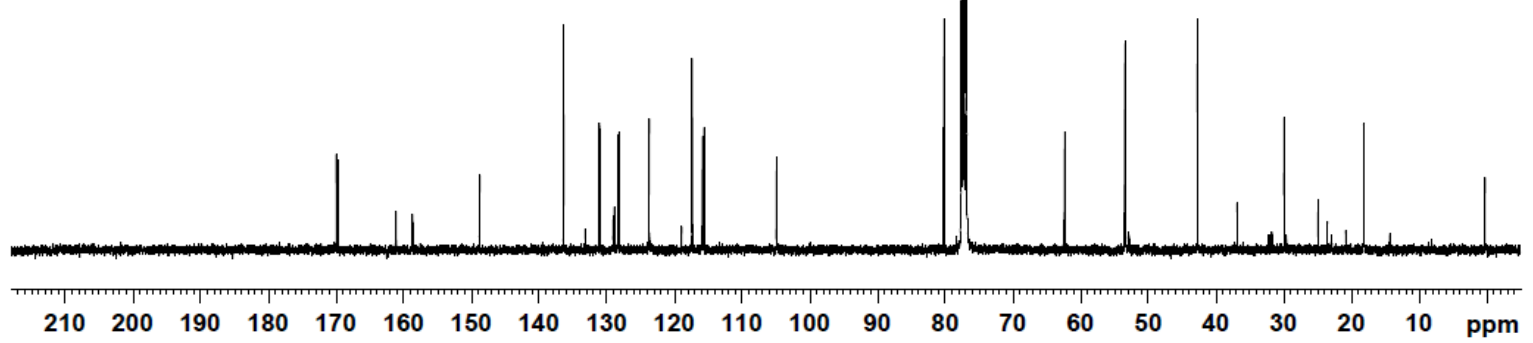




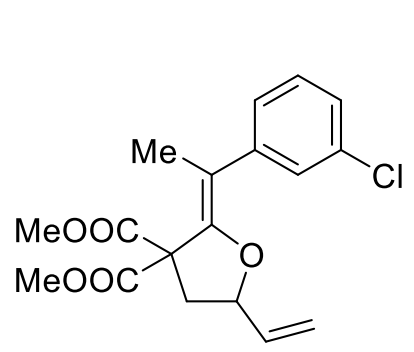

$( \pm)-3 g$

${ }^{1} \mathrm{H} N \mathrm{NMR}$

$\mathrm{CDCl}_{3}, 400 \mathrm{MHz}$

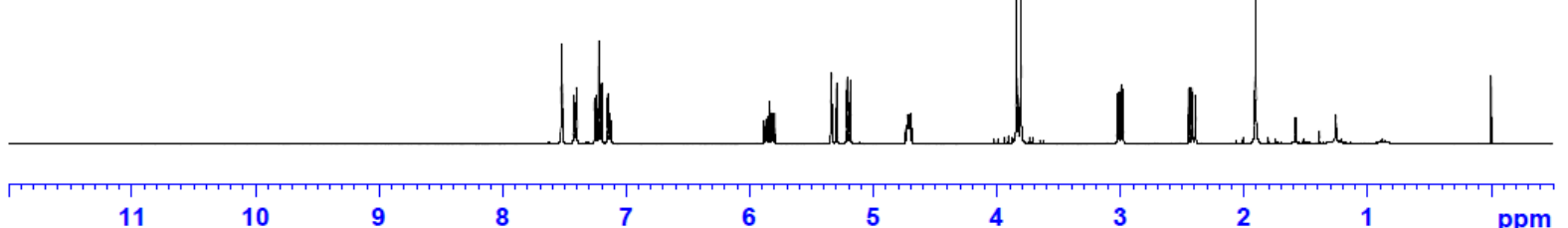

$\begin{array}{llllllllllll}11 & 10 & 9 & 8 & 7 & 6 & 5 & 4 & 3 & 2 & 1 & \mathrm{ppm}\end{array}$

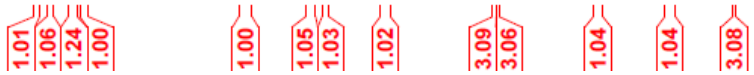

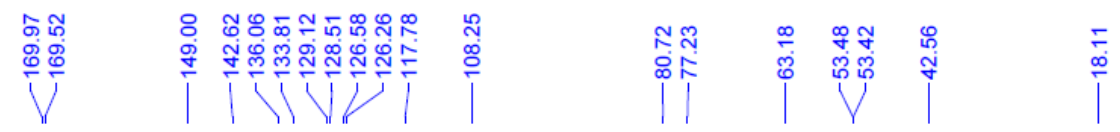<smiles>C=CC1CC(C(C)=O)(C(C)=O)C(=C(C)c2cccc(Cl)c2)O1</smiles>

$( \pm)-3 g$

${ }^{13} \mathrm{C}$ NMR

$\mathrm{CDCl}_{3}, 100 \mathrm{MHz}$

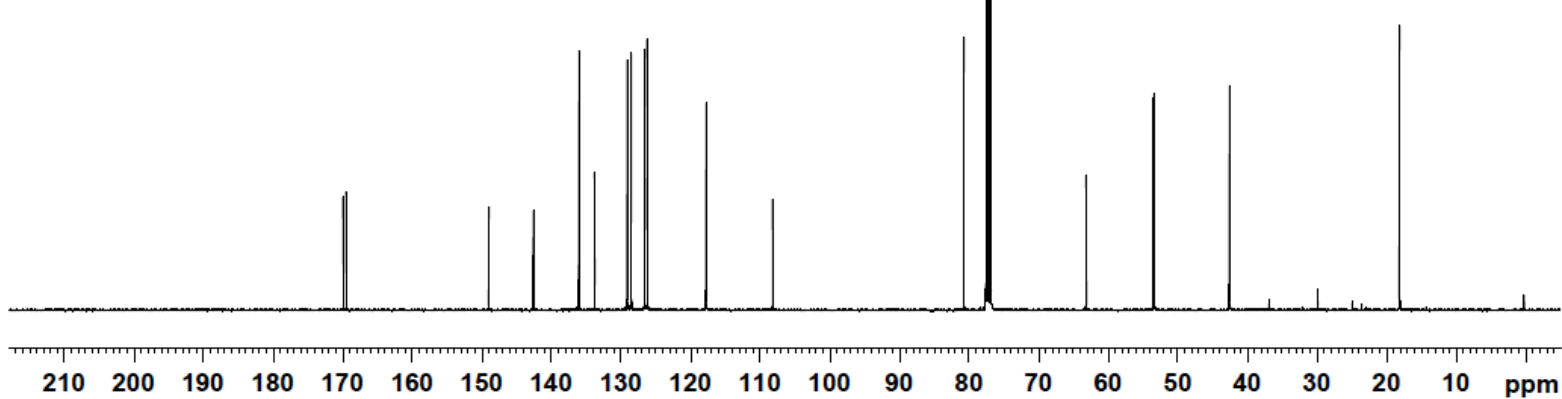




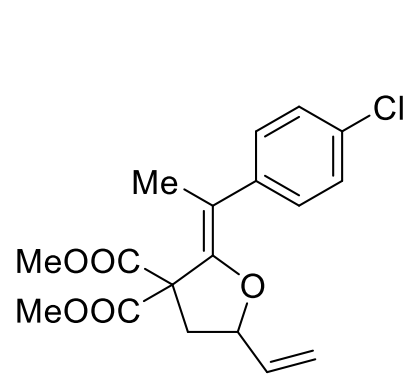

( \pm )-3h

${ }^{1} \mathrm{H}$ NMR

$\mathrm{CDCl}_{3}, 400 \mathrm{MHz}$
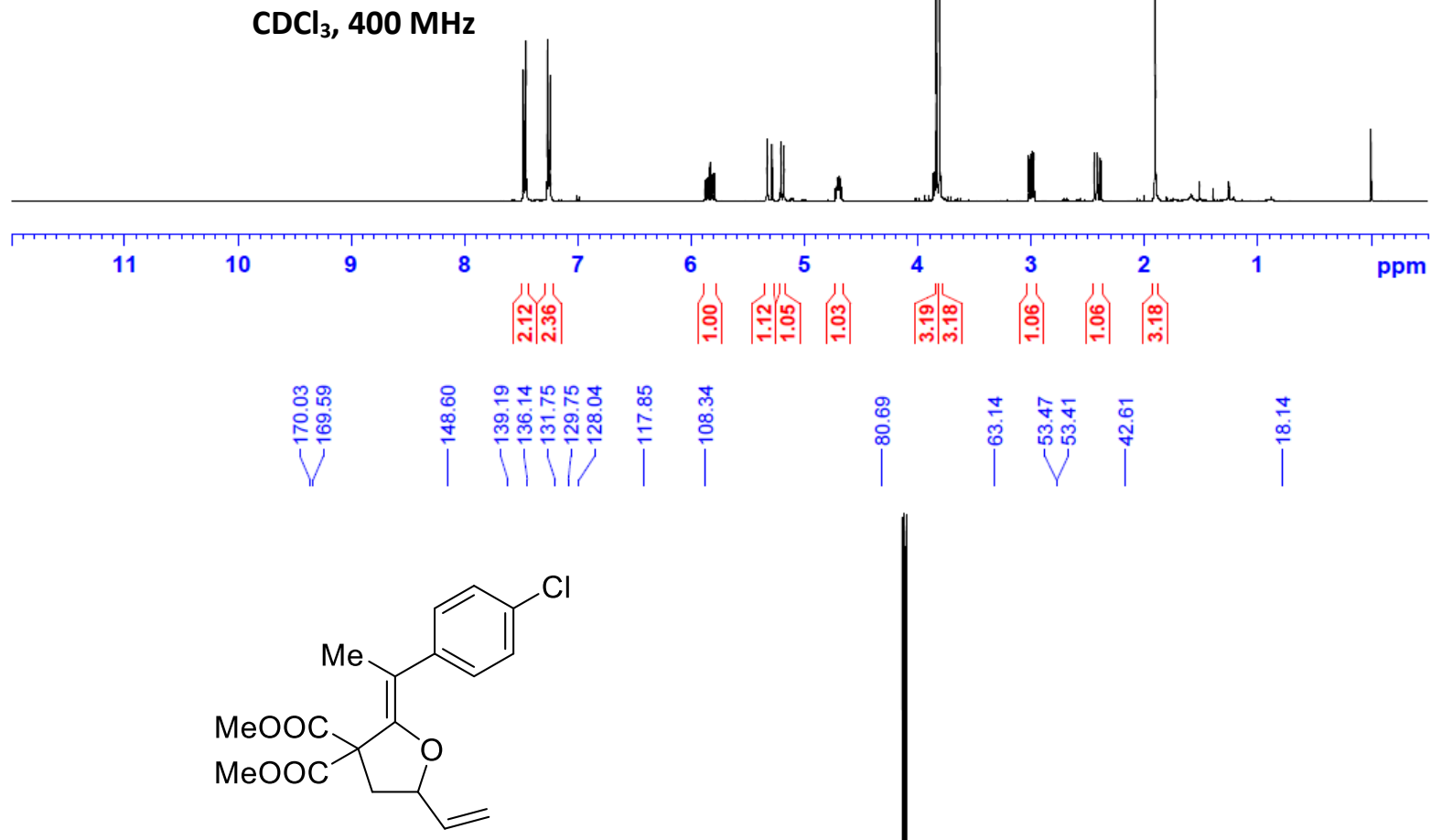

( \pm )-3h

${ }^{13} \mathrm{C}$ NMR

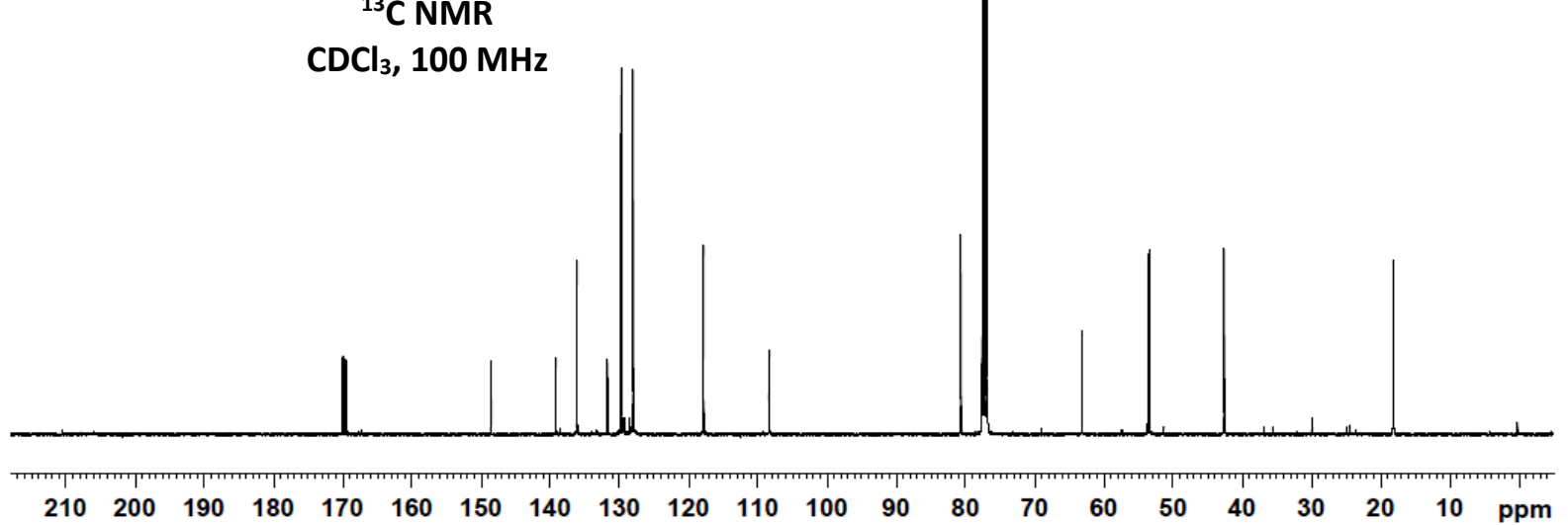




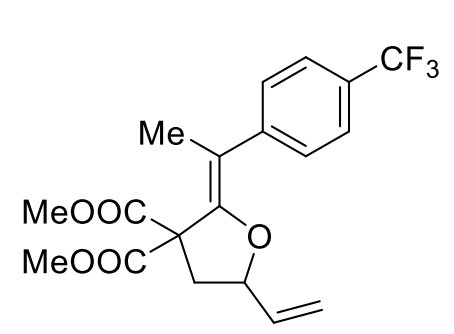

( \pm )-3i

${ }^{1} \mathrm{H}$ NMR

$\mathrm{CDCl}_{3}, 400 \mathrm{MHz}$

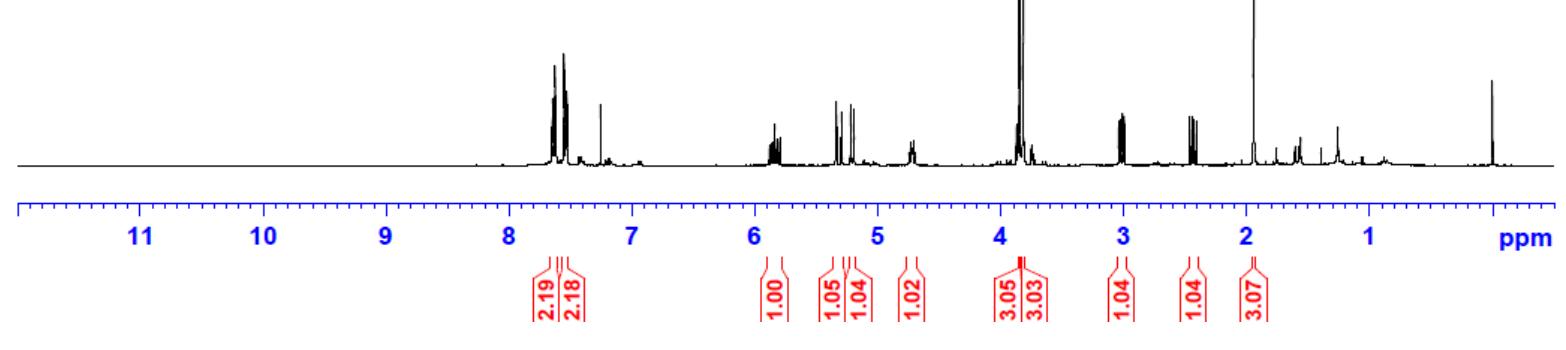

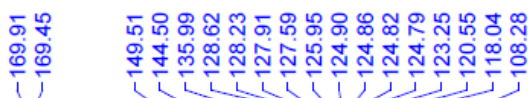

(

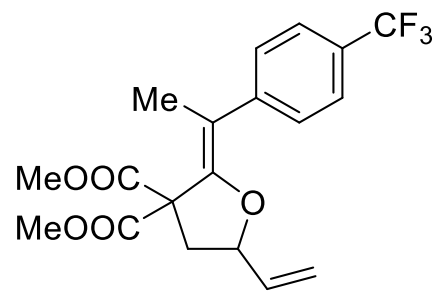

$( \pm)-3 \mathbf{i}$

${ }^{13} \mathrm{C}$ NMR

$\mathrm{CDCl}_{3}, 100 \mathrm{MHz}$

$\begin{array}{lllllllllllllllllllllll}210 & 200 & 190 & 180 & 170 & 160 & 150 & 140 & 130 & 120 & 110 & 100 & 90 & 80 & 70 & 60 & 50 & 40 & 30 & 20 & 10 & \text { ppm }\end{array}$ 


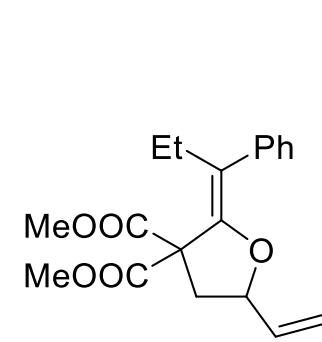

$( \pm)-3 \mathbf{j}$

${ }^{1} \mathrm{H} N \mathrm{NMR}$

$\mathrm{CDCl}_{3}, 400 \mathrm{MHz}$

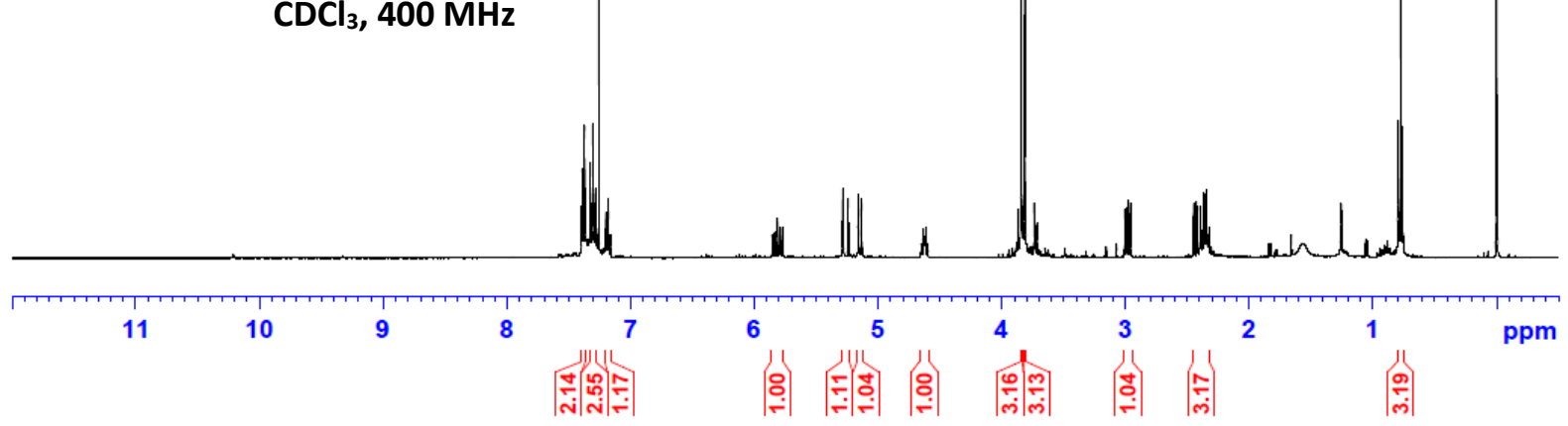

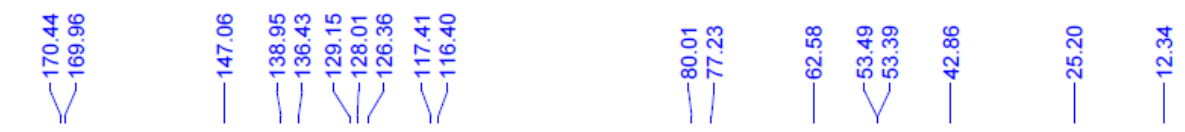

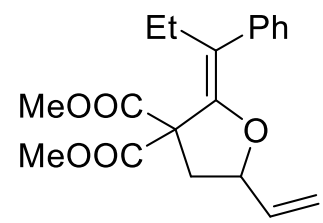

$( \pm)-3 \mathbf{j}$

${ }^{13} \mathrm{C}$ NMR

$\mathrm{CDCl}_{3}, 100 \mathrm{MHz}$

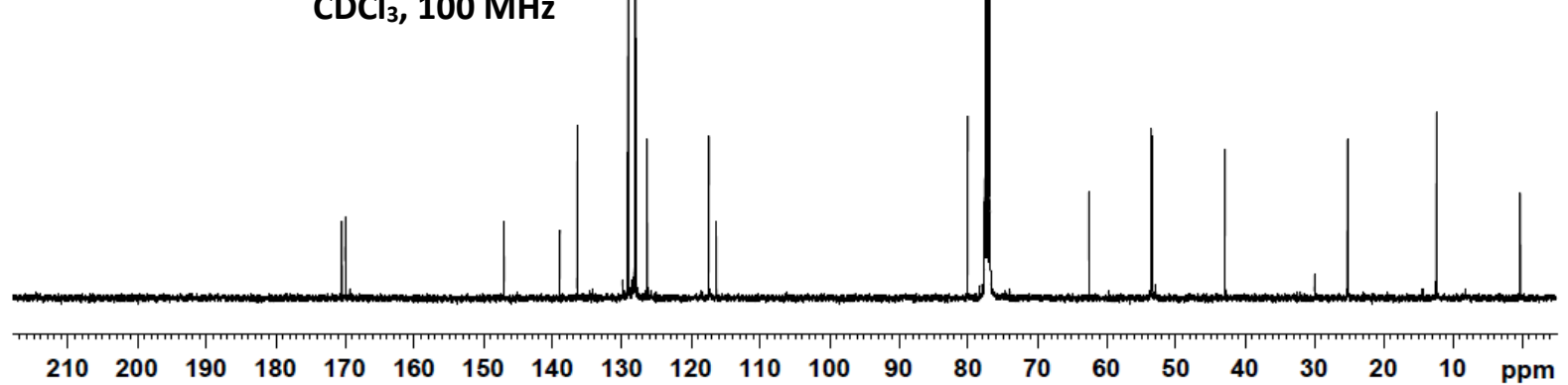




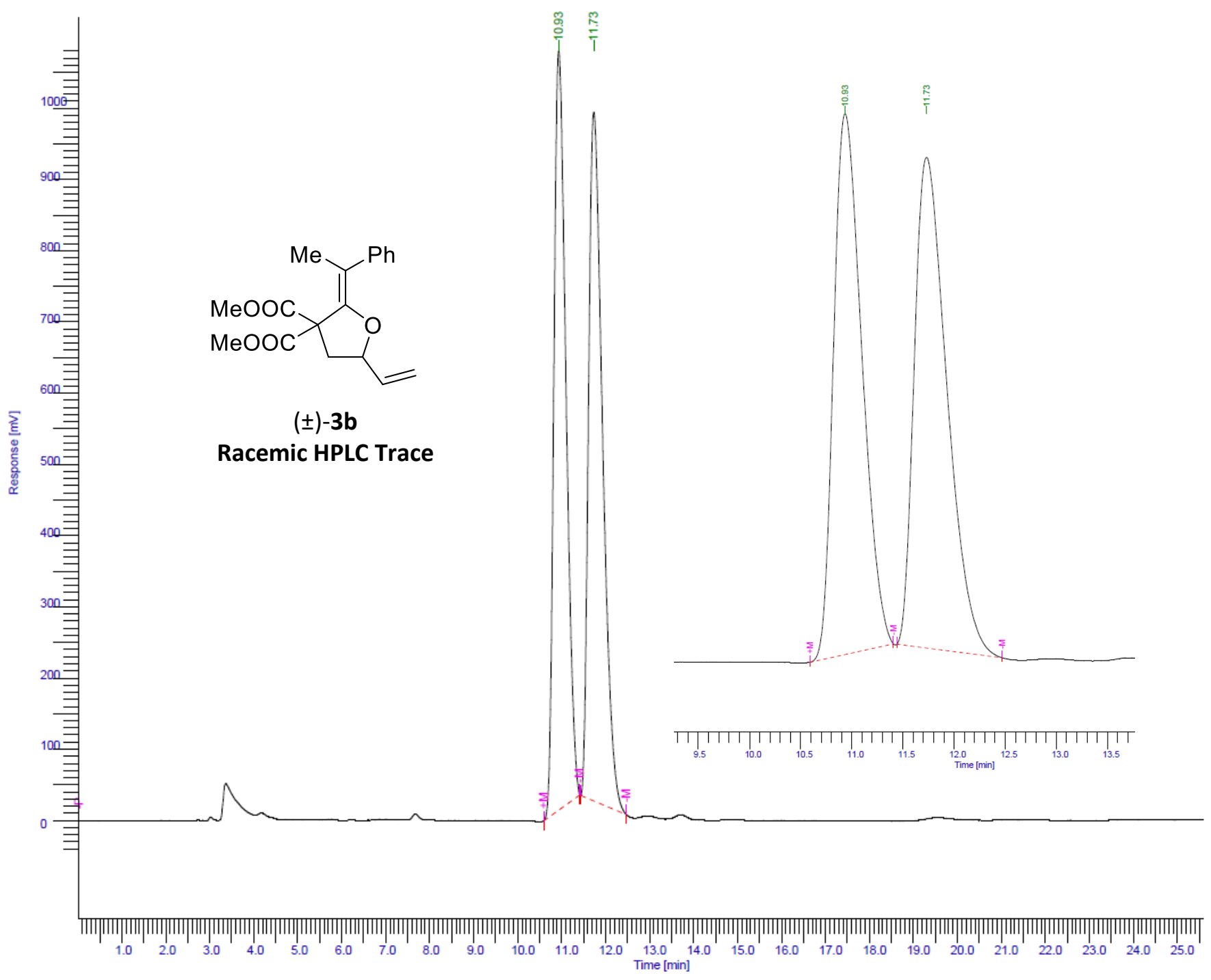

\begin{tabular}{|c|c|c|c|c|c|c|c|}
\hline $\begin{array}{l}\text { Peak } \\
\quad \#\end{array}$ & $\begin{array}{l}\text { Time } \\
\text { [min] }\end{array}$ & $\begin{array}{l}\text { Area } \\
{[\mu \mathrm{V} \cdot \mathrm{s}]}\end{array}$ & $\begin{array}{l}\text { Height } \\
{[\mu \mathrm{V}]}\end{array}$ & $\begin{array}{c}\text { Area } \\
{[\%]}\end{array}$ & $\begin{array}{c}\text { Norm. Area } \\
{[\%]}\end{array}$ & $B L$ & $\begin{array}{c}\text { Area/Height } \\
\text { [s] }\end{array}$ \\
\hline & \multirow{3}{*}{$\begin{array}{l}10.934 \\
11.731\end{array}$} & 20413834.17 & $1.07 e+06$ & 48.79 & 48.79 & \multirow{3}{*}{$\begin{array}{l}{ }^{*} \mathrm{BB} \\
{ }^{*} \mathrm{BB}\end{array}$} & \multirow{3}{*}{$\begin{array}{l}19.1459 \\
22.1499\end{array}$} \\
\hline & & 21423815.43 & 967219.86 & 51.21 & 51.21 & & \\
\hline & & 41837649.60 & $2.03 e+06$ & 100.00 & 100.00 & & \\
\hline
\end{tabular}




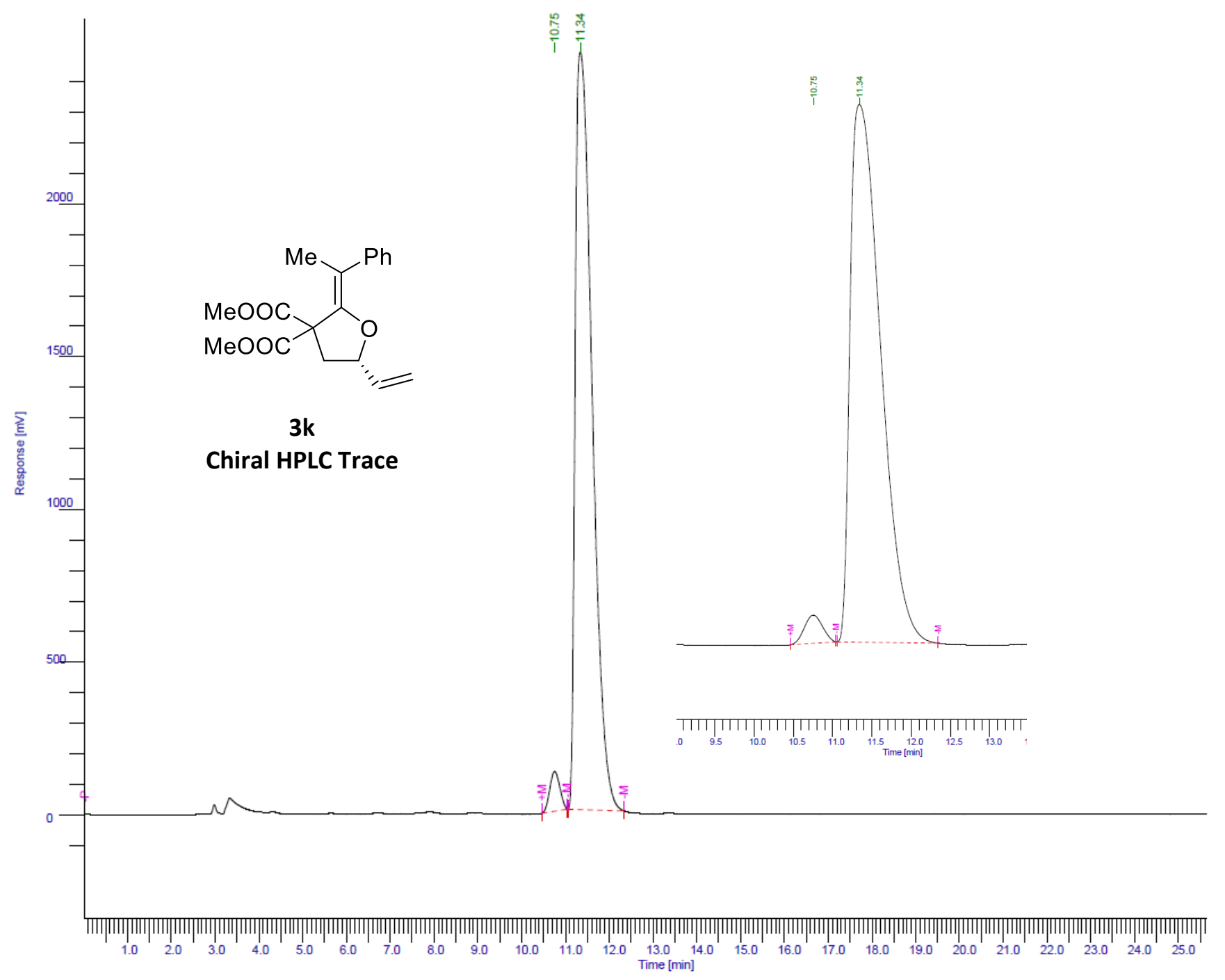

\begin{tabular}{|c|c|c|c|c|c|c|c|}
\hline $\begin{array}{c}\text { Peak } \\
\#\end{array}$ & $\begin{array}{l}\text { Time } \\
\text { [min] }\end{array}$ & $\begin{array}{l}\text { Area } \\
{[\mu \mathrm{V} \cdot \mathrm{s}]}\end{array}$ & $\begin{array}{l}\text { Height } \\
{[\mu \mathrm{V}]}\end{array}$ & $\begin{array}{c}\text { Area } \\
{[\%]}\end{array}$ & $\begin{array}{c}\text { Norm. Area } \\
{[\%]}\end{array}$ & $B L$ & $\begin{array}{c}\text { Area/Height } \\
\text { [s] }\end{array}$ \\
\hline \multirow{3}{*}{2} & 10.754 & 2094146.46 & 130492.57 & 3.04 & 3.04 & ${ }^{*} \mathrm{BB}$ & 16.0480 \\
\hline & 11.340 & 66728712.41 & $2.48 e+06$ & 96.96 & 96.96 & ${ }^{*} \mathrm{BB}$ & 26.8939 \\
\hline & & 68822858.87 & $2.61 e+06$ & 100.00 & 100.00 & & \\
\hline
\end{tabular}




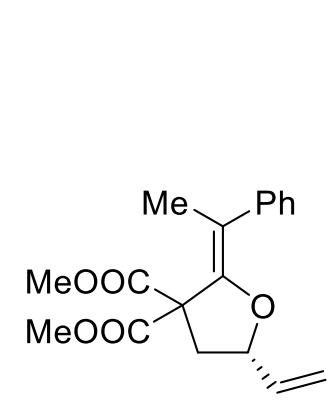

3k

${ }^{1} \mathrm{H}$ NMR

$\mathrm{CDCl}_{3}, 400 \mathrm{MHz}$

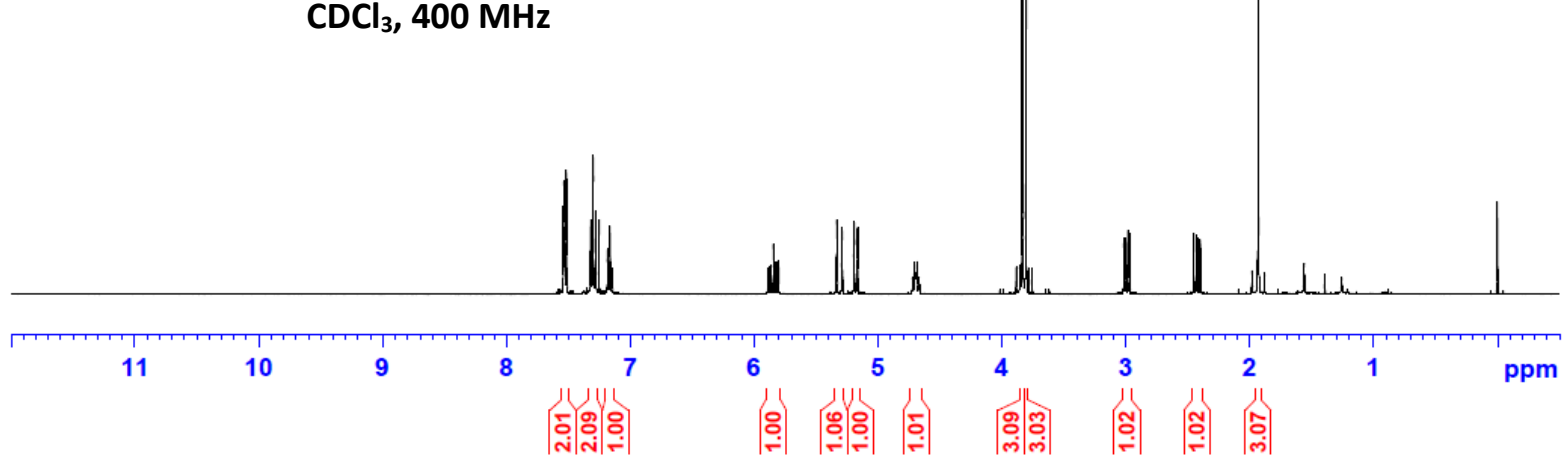

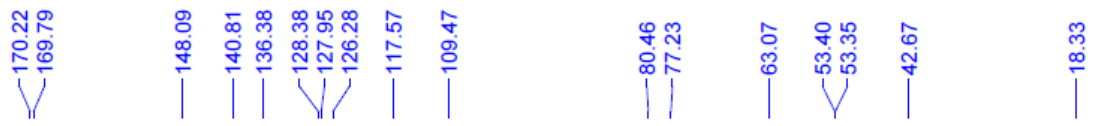

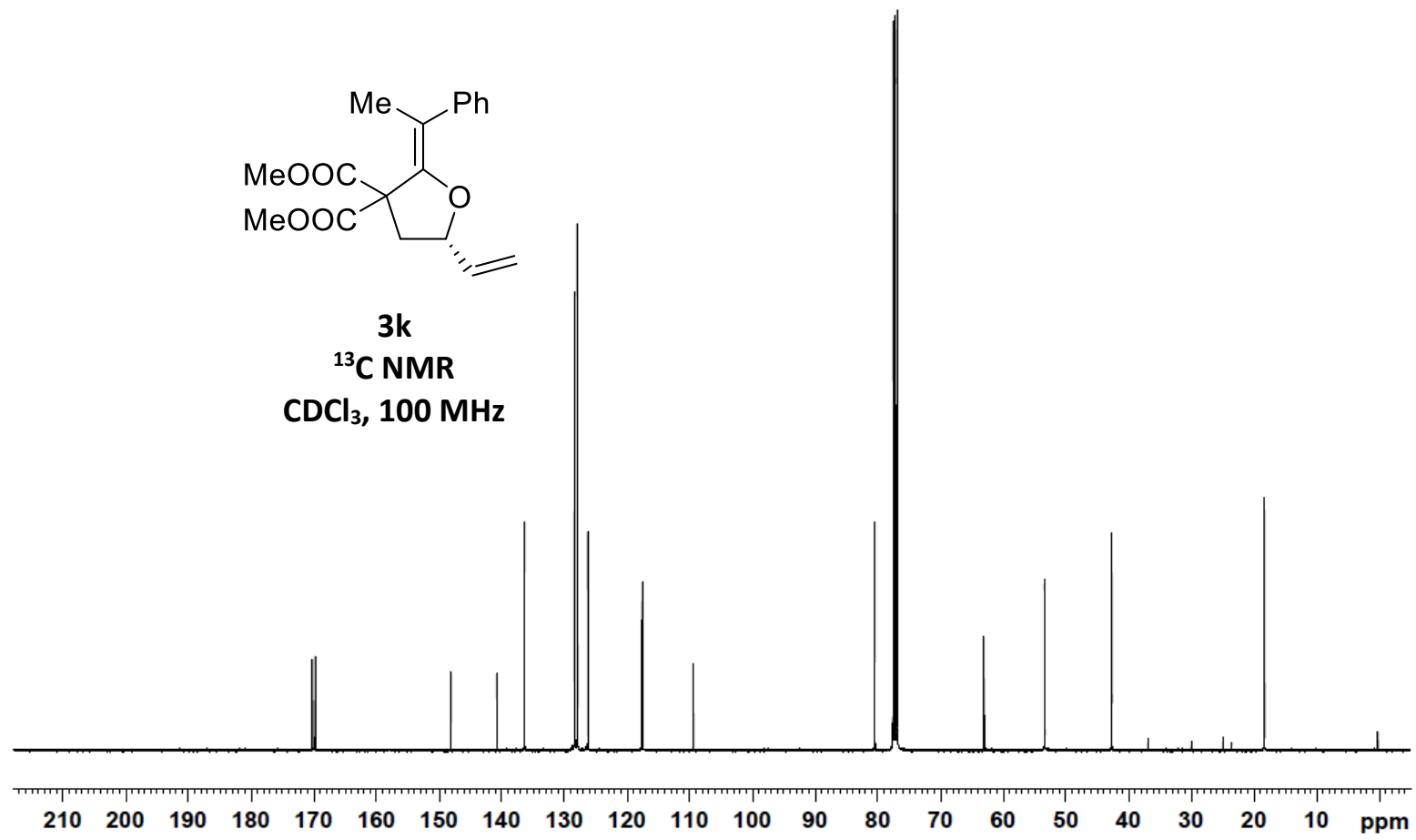




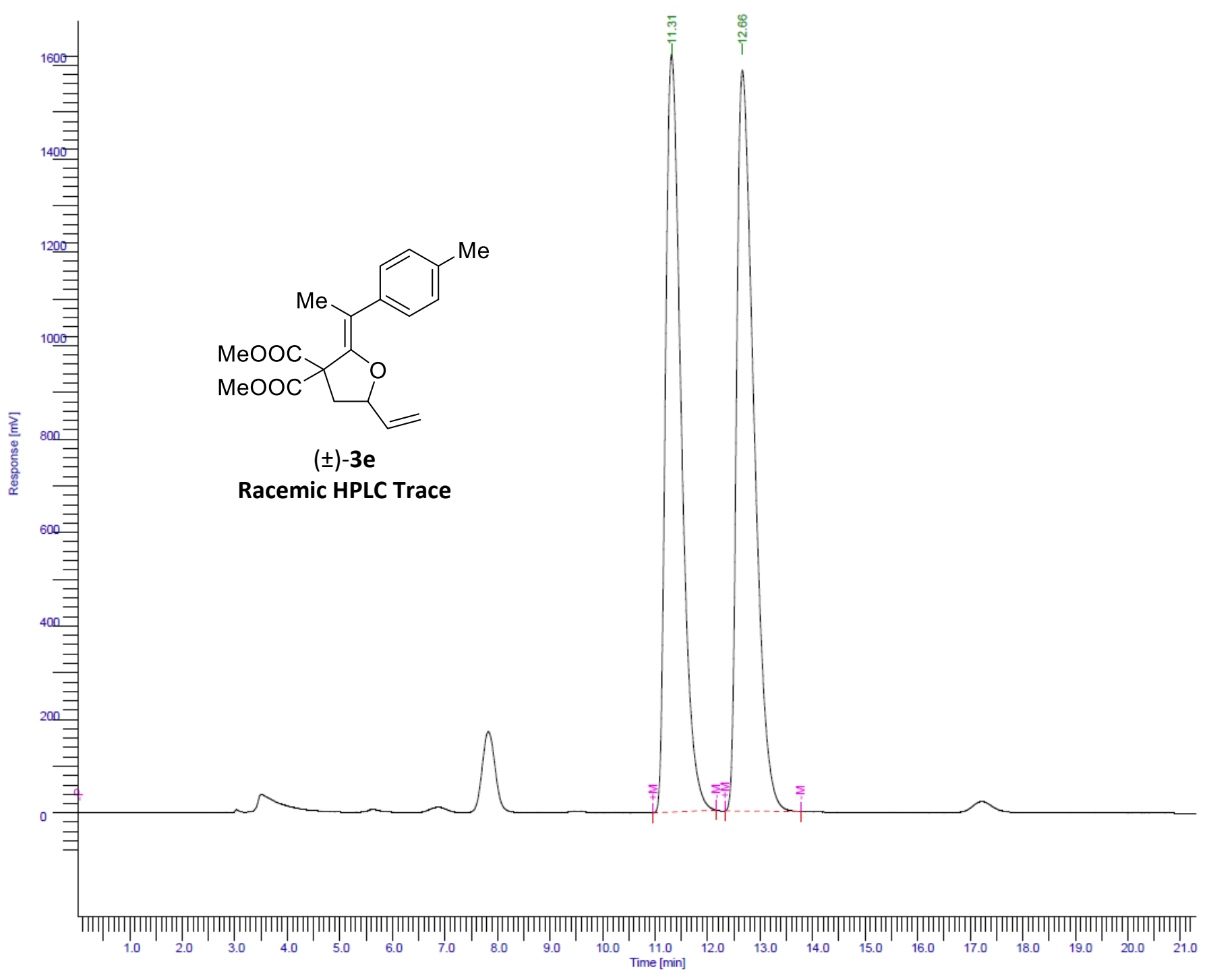

\begin{tabular}{|c|c|c|c|c|c|c|c|}
\hline $\begin{array}{l}\text { Peak } \\
\#\end{array}$ & $\begin{array}{l}\text { Time } \\
\text { [min] }\end{array}$ & $\begin{array}{l}\text { Area } \\
{[\mu \vee \cdot s]}\end{array}$ & $\begin{array}{l}\text { Height } \\
{[\mu \mathrm{V}]}\end{array}$ & $\begin{array}{c}\text { Area } \\
{[\%]}\end{array}$ & $\begin{array}{c}\text { Norm. Area } \\
{[\%]}\end{array}$ & $B L$ & $\begin{array}{c}\text { Area/Height } \\
\text { [s] }\end{array}$ \\
\hline & 11.314 & 33807224.15 & $1.62 e+06$ & 48.25 & 48.25 & ${ }^{*} \mathrm{BB}$ & 20.8452 \\
\hline & 12.662 & 36263506.84 & $1.59 e+06$ & 51.75 & 51.75 & ${ }^{*} \mathrm{BB}$ & 22.8748 \\
\hline & & 70070730.99 & $3.21 e+06$ & 100.00 & 100.00 & & \\
\hline
\end{tabular}




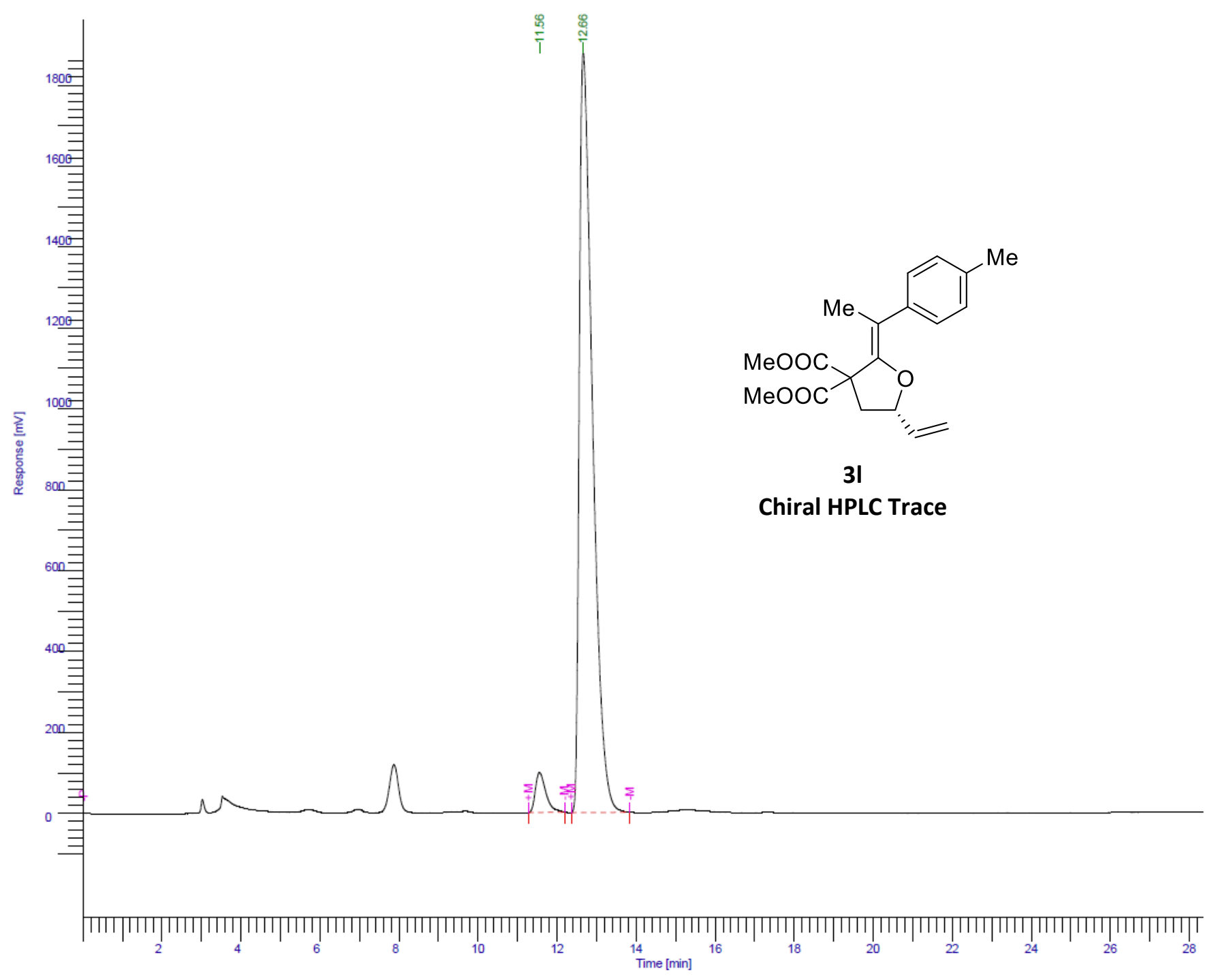

\begin{tabular}{|c|c|c|c|c|c|c|c|}
\hline $\begin{array}{l}\text { Peak } \\
\quad \#\end{array}$ & $\begin{array}{l}\text { Time } \\
\text { [min] }\end{array}$ & $\begin{array}{l}\text { Area } \\
{[\mu \mathrm{V} \cdot \mathrm{s}]}\end{array}$ & $\begin{array}{l}\text { Height } \\
{[\mu \mathrm{V}]}\end{array}$ & $\begin{array}{c}\text { Area } \\
{[\%]}\end{array}$ & $\begin{array}{c}\text { Norm. Area } \\
{[\%]}\end{array}$ & $B L$ & $\begin{array}{c}\text { Area/Height } \\
\text { [s] }\end{array}$ \\
\hline \multirow{3}{*}{$\begin{array}{l}1 \\
2\end{array}$} & 11.556 & 1831121.79 & 98934.34 & 4.03 & 4.03 & ${ }^{*} \mathrm{BB}$ & 18.5085 \\
\hline & 12.664 & 43635795.83 & $1.88 e+06$ & 95.97 & 95.97 & ${ }^{*} \mathrm{BB}$ & 23.2371 \\
\hline & & 45466917.62 & $1.98 e+06$ & 100.00 & 100.00 & & \\
\hline
\end{tabular}




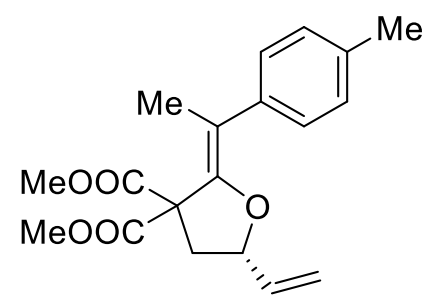

31

${ }^{1} \mathrm{H}$ NMR

$\mathrm{CDCl}_{3}, 400 \mathrm{MHz}$

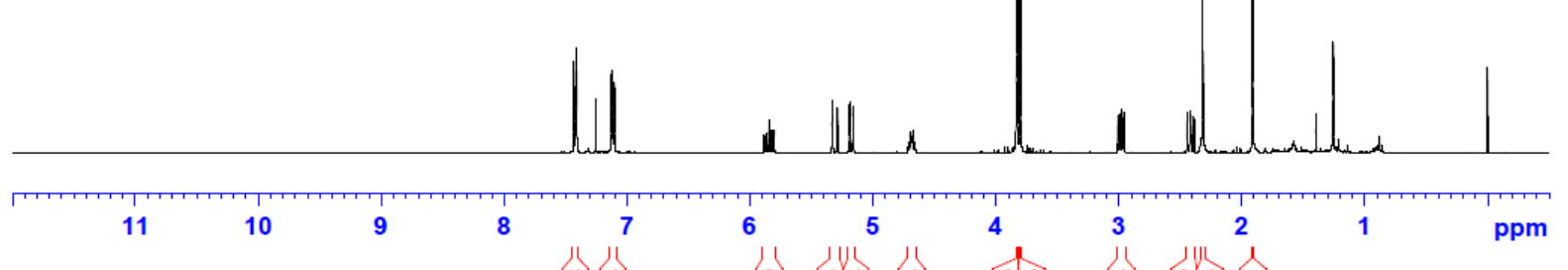

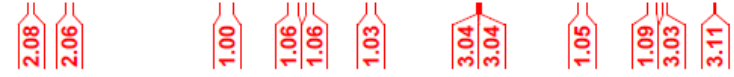

\begin{tabular}{|c|c|c|c|c|c|c|c|}
\hline 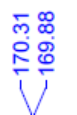 & 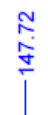 & 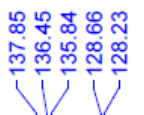 & 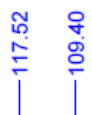 & 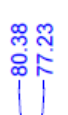 & $\begin{array}{l}\overline{0} \\
\text { i. }\end{array}$ & 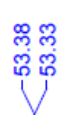 & ำ \\
\hline
\end{tabular}

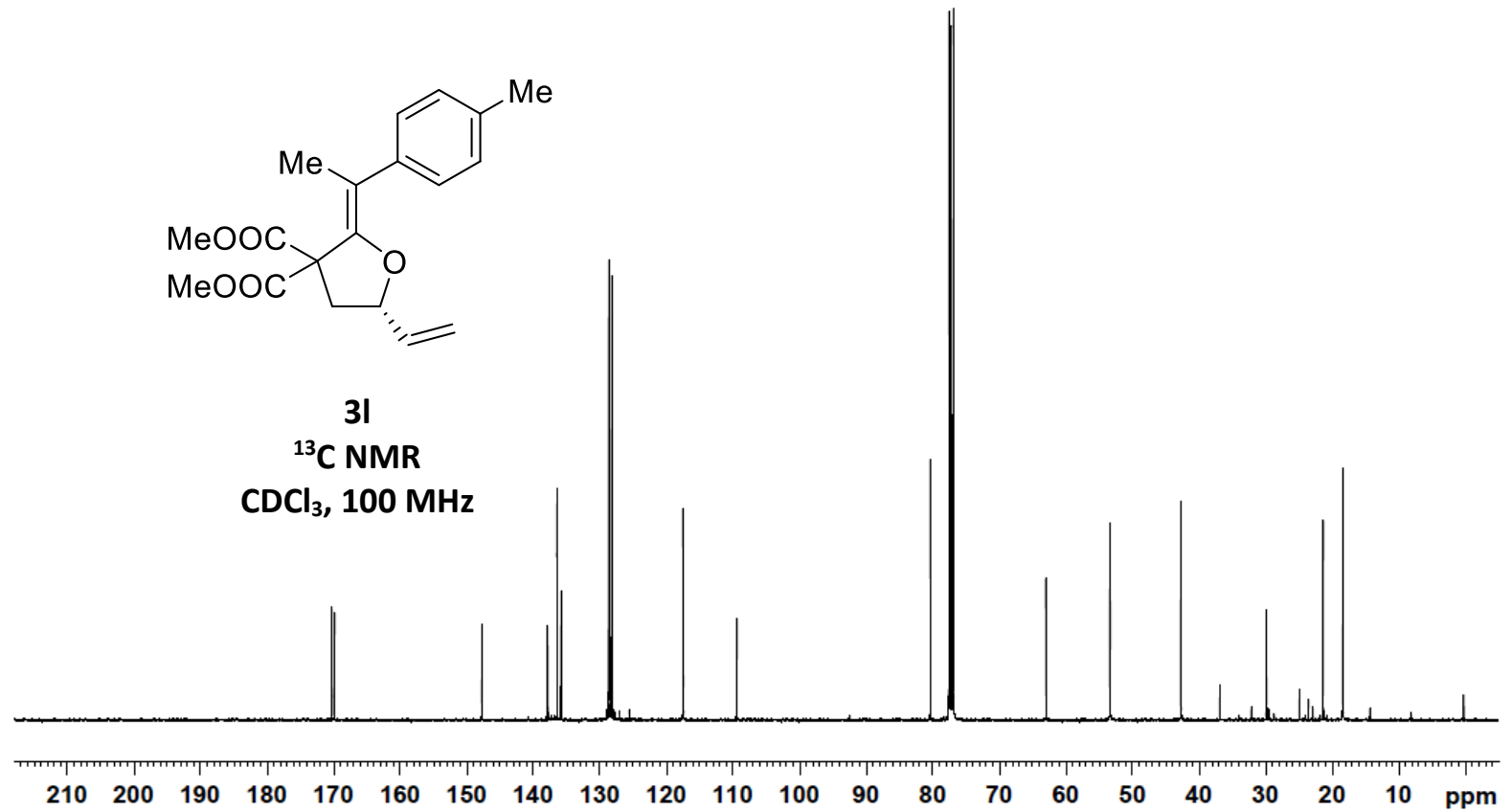




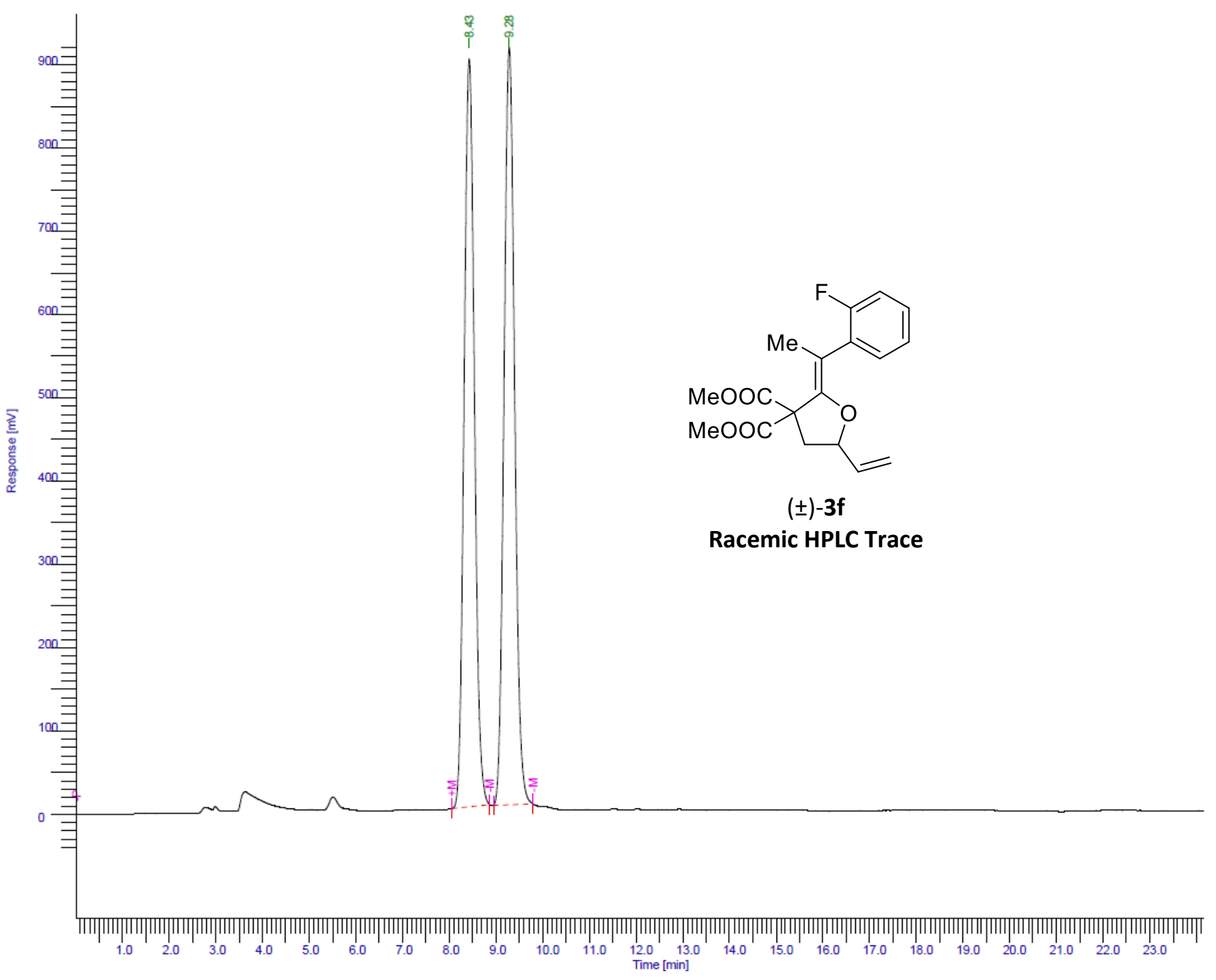

\begin{tabular}{|c|c|c|c|c|c|c|c|}
\hline $\begin{array}{l}\text { Peak } \\
\quad \#\end{array}$ & $\begin{array}{l}\text { Time } \\
\text { [min] }\end{array}$ & $\begin{array}{l}\text { Area } \\
{[\mu \mathrm{V} \cdot \mathrm{s}]}\end{array}$ & $\begin{array}{l}\text { Height } \\
{[\mu \mathrm{V}]}\end{array}$ & $\begin{array}{c}\text { Area } \\
{[\%]}\end{array}$ & $\begin{array}{c}\text { Norm. Area } \\
{[\%]}\end{array}$ & $B L$ & $\begin{array}{c}\text { Area/Height } \\
\text { [s] }\end{array}$ \\
\hline & 8.425 & 13756882.77 & 897729.05 & 48.95 & 48.95 & ${ }^{*} \mathrm{BB}$ & 15.3241 \\
\hline & 9.282 & 14347856.62 & 909410.99 & 51.05 & 51.05 & ${ }^{*} \mathrm{BB}$ & 15.7771 \\
\hline & & 28104739.39 & $1.81 e+06$ & 100.00 & 100.00 & & \\
\hline
\end{tabular}




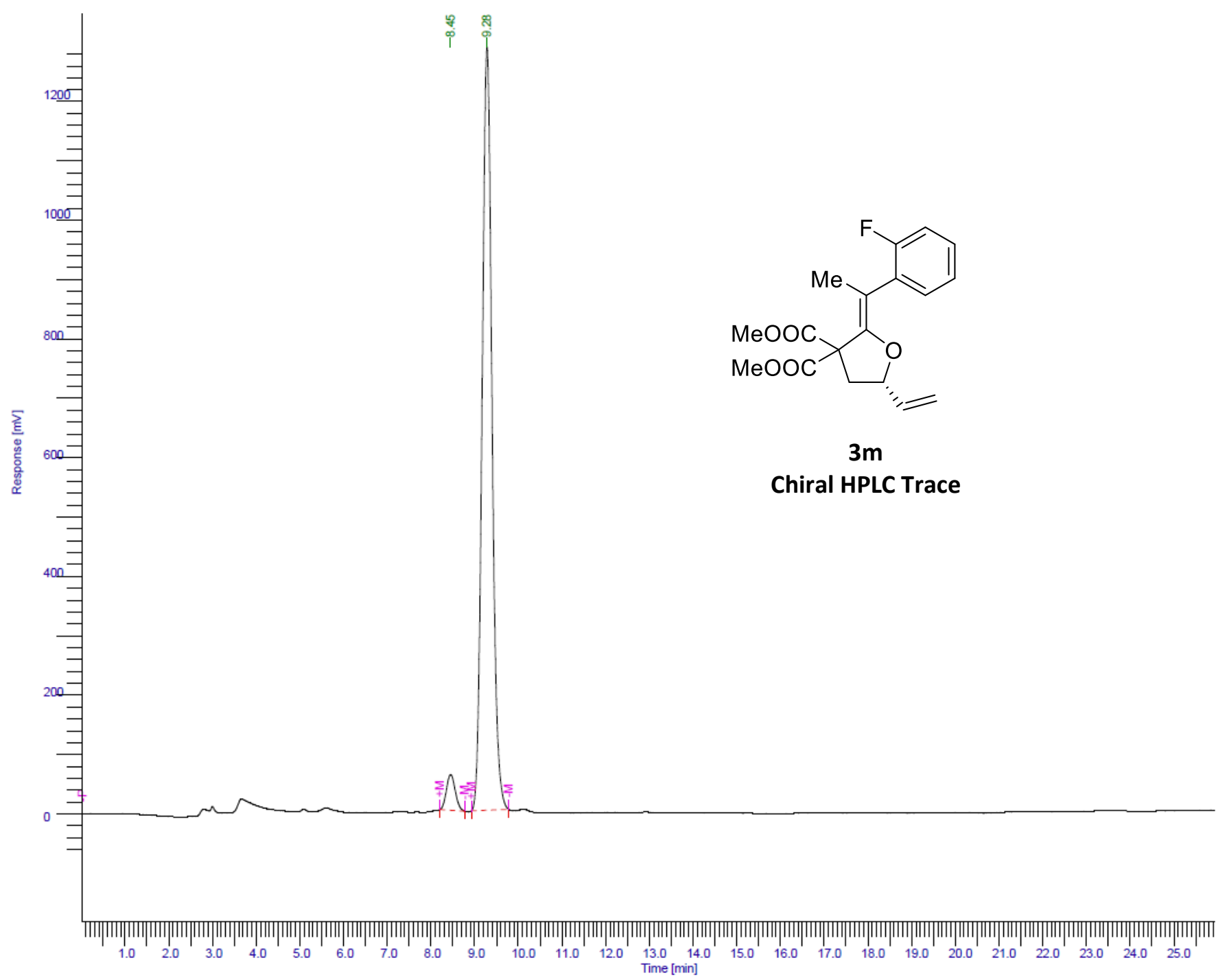

\begin{tabular}{|c|c|c|c|c|c|c|c|}
\hline $\begin{array}{c}\text { Peak } \\
\#\end{array}$ & $\begin{array}{l}\text { Time } \\
\text { [min] }\end{array}$ & $\begin{array}{l}\text { Area } \\
{[\mu \mathrm{V} \cdot \mathrm{s}]}\end{array}$ & $\begin{array}{l}\text { Height } \\
{[\mu \mathrm{V}]}\end{array}$ & $\begin{array}{l}\text { Area } \\
{[\%]}\end{array}$ & $\begin{array}{c}\text { Norm. Area } \\
{[\%]}\end{array}$ & $\mathrm{BL}$ & $\begin{array}{c}\text { Area/Height } \\
{[\mathrm{s}]}\end{array}$ \\
\hline & 8.449 & 844062.06 & 60338.75 & 4.02 & 4.02 & ${ }^{*} \mathrm{BB}$ & 13.9887 \\
\hline & 9.281 & 20136374.76 & $1.29 e+06$ & 95.98 & 95.98 & ${ }^{*} \mathrm{BB}$ & 15.6607 \\
\hline & & 20980436.8 & $1.35 e+06$ & 100.00 & 100.00 & & \\
\hline
\end{tabular}




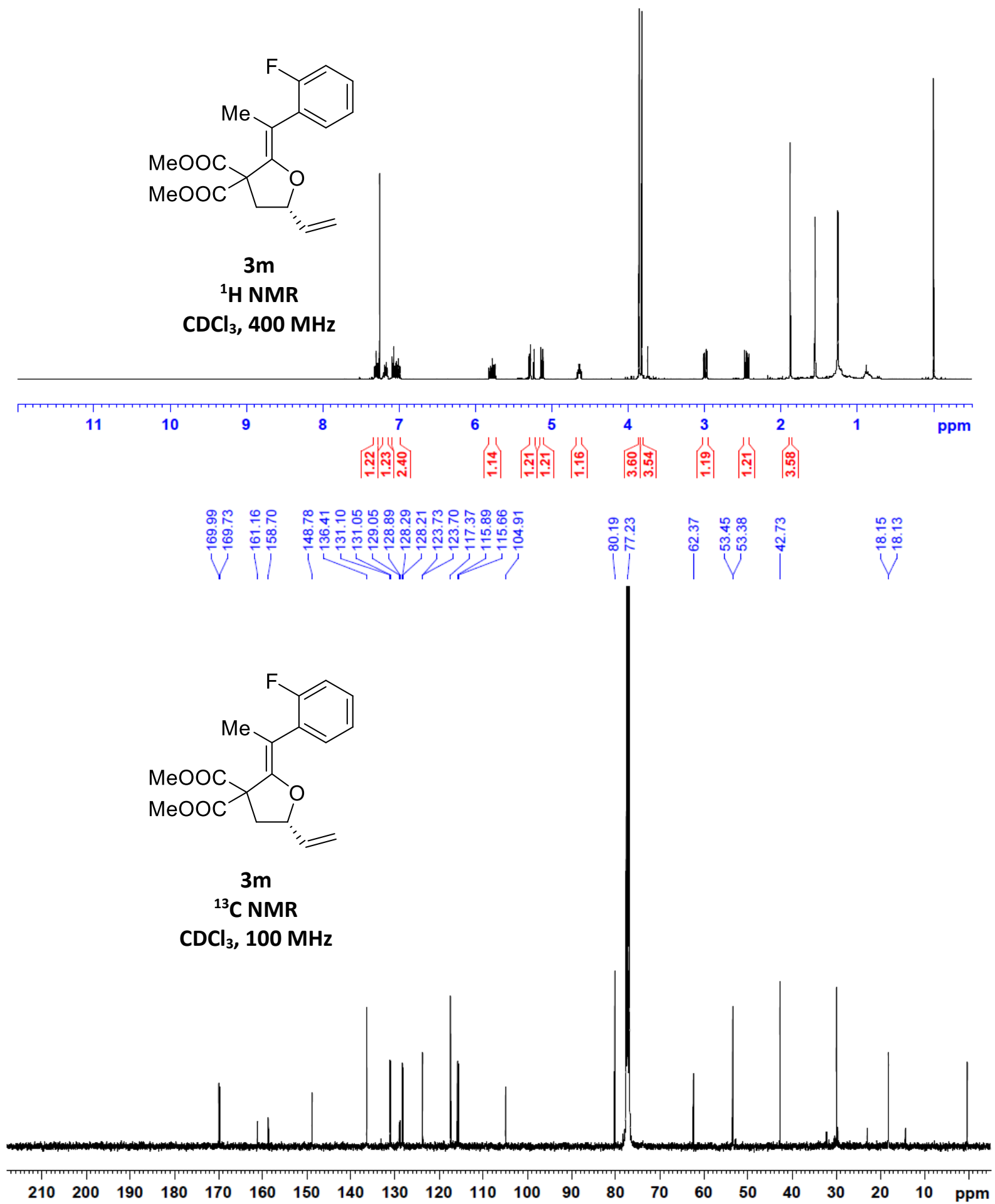




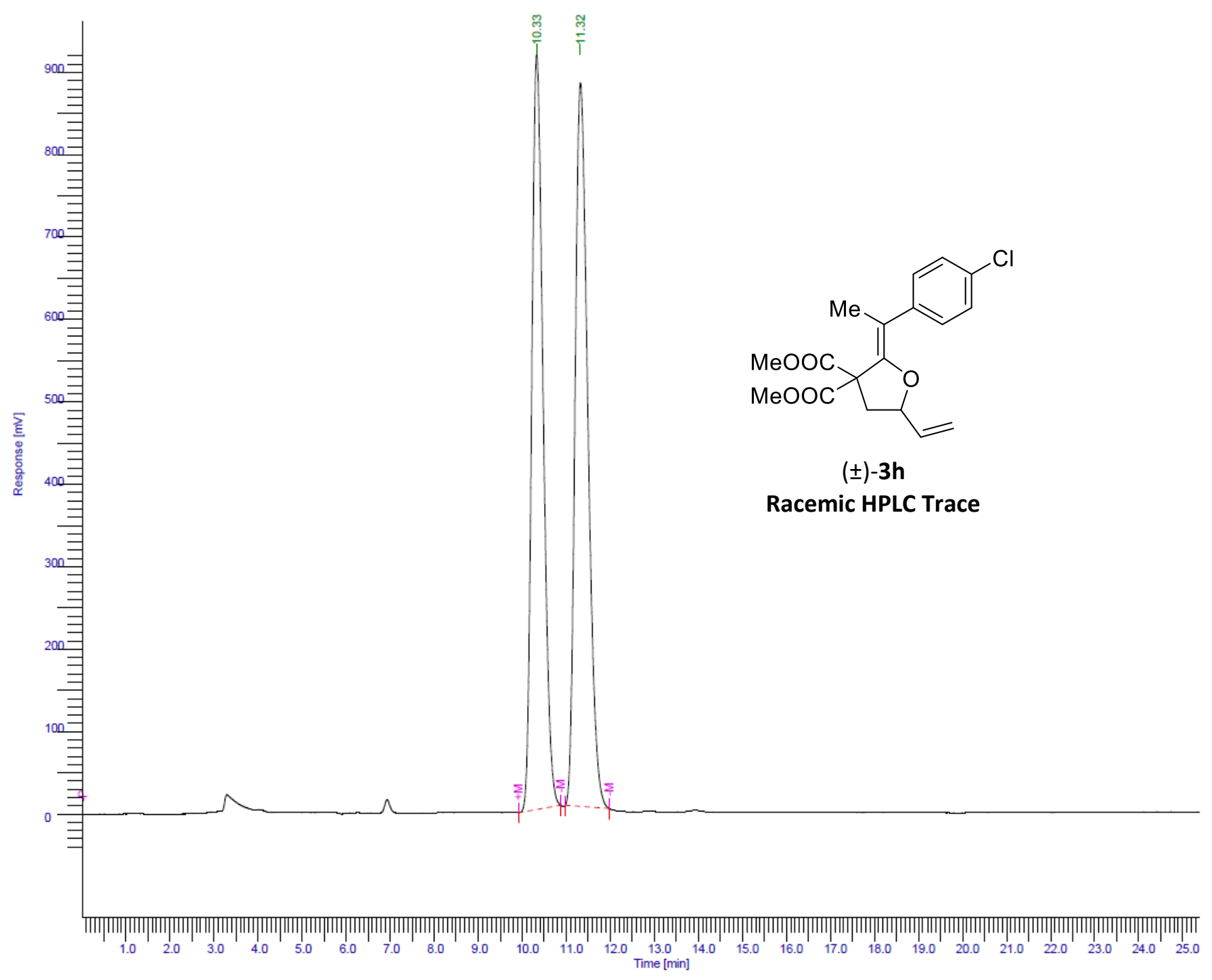

\begin{tabular}{|c|c|c|c|c|c|c|c|}
\hline $\begin{array}{l}\text { Peak } \\
\quad \#\end{array}$ & $\begin{array}{l}\text { Time } \\
\text { [min] }\end{array}$ & $\begin{array}{l}\text { Area } \\
{[\mu \mathrm{V} \cdot \mathrm{s}]}\end{array}$ & $\begin{array}{l}\text { Height } \\
{[\mu \mathrm{V}]}\end{array}$ & $\begin{array}{c}\text { Area } \\
{[\%]}\end{array}$ & $\begin{array}{c}\text { Norm. Area } \\
{[\%]}\end{array}$ & $\mathrm{BL}$ & $\begin{array}{c}\text { Area/Height } \\
\text { [s] }\end{array}$ \\
\hline & 10.328 & 16457294.12 & 916388.54 & 48.17 & 48.17 & ${ }^{*} \mathrm{BB}$ & 17.9589 \\
\hline & 11.322 & 17710465.80 & 878047.06 & 51.83 & 51.83 & ${ }^{*} \mathrm{BB}$ & 20.1703 \\
\hline & & 34167759.92 & $1.79 e+06$ & 100.00 & 100.00 & & \\
\hline
\end{tabular}




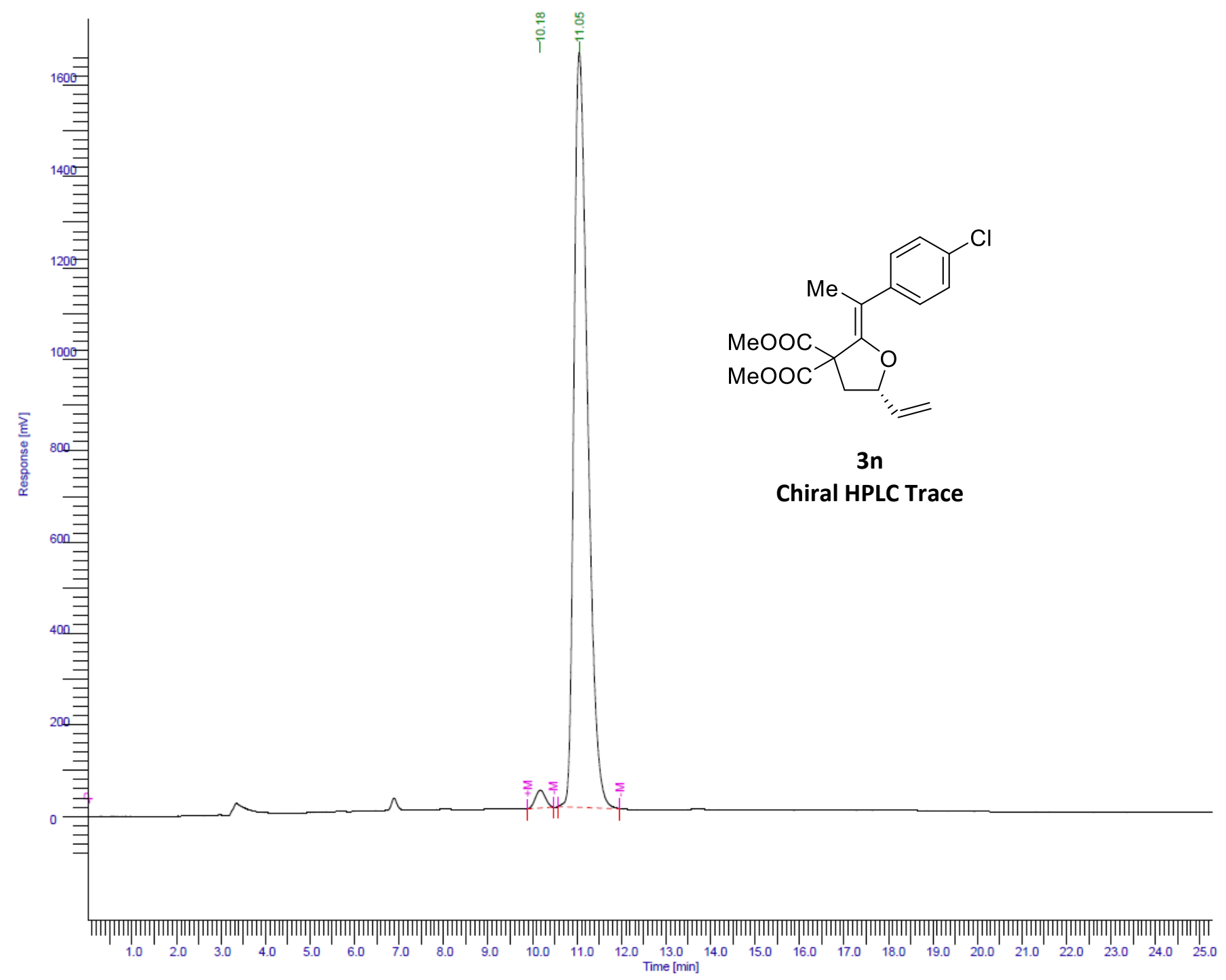

\begin{tabular}{|c|c|c|c|c|c|c|c|}
\hline $\begin{array}{l}\text { Peak } \\
\quad \#\end{array}$ & $\begin{array}{l}\text { Time } \\
\text { [min] }\end{array}$ & $\begin{array}{l}\text { Area } \\
{[\mu \mathrm{V} \cdot \mathrm{s}]}\end{array}$ & $\begin{array}{l}\text { Height } \\
{[\mu \mathrm{V}]}\end{array}$ & $\begin{array}{c}\text { Area } \\
{[\%]}\end{array}$ & $\begin{array}{c}\text { Norm. Area } \\
{[\%]}\end{array}$ & $B L$ & $\begin{array}{c}\text { Area/Height } \\
{[\mathrm{s}]}\end{array}$ \\
\hline & 10.176 & 612970.00 & 39328.72 & 1.70 & 1.70 & ${ }^{*} \mathrm{BB}$ & 15.5858 \\
\hline & 11.051 & 35373611.71 & $1.65 e+06$ & 98.30 & 98.30 & ${ }^{*} \mathrm{BB}$ & 21.4038 \\
\hline & & 35986581.71 & $1.69 e+06$ & 100.00 & 100.00 & & \\
\hline
\end{tabular}




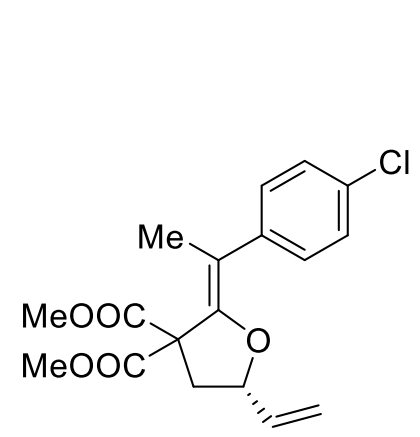

$3 n$

${ }^{1} \mathrm{H}$ NMR

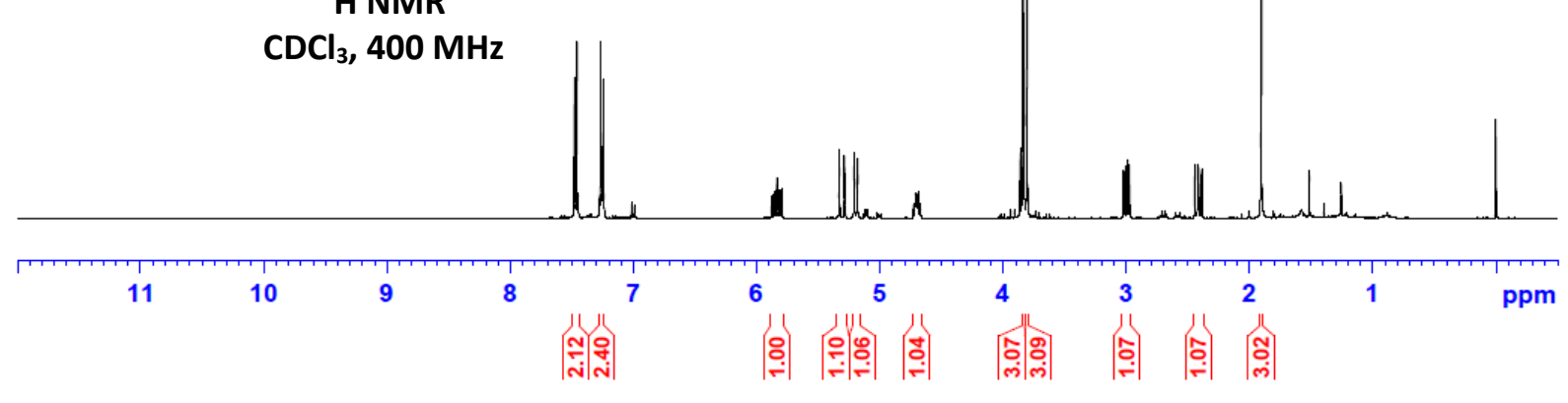
隹

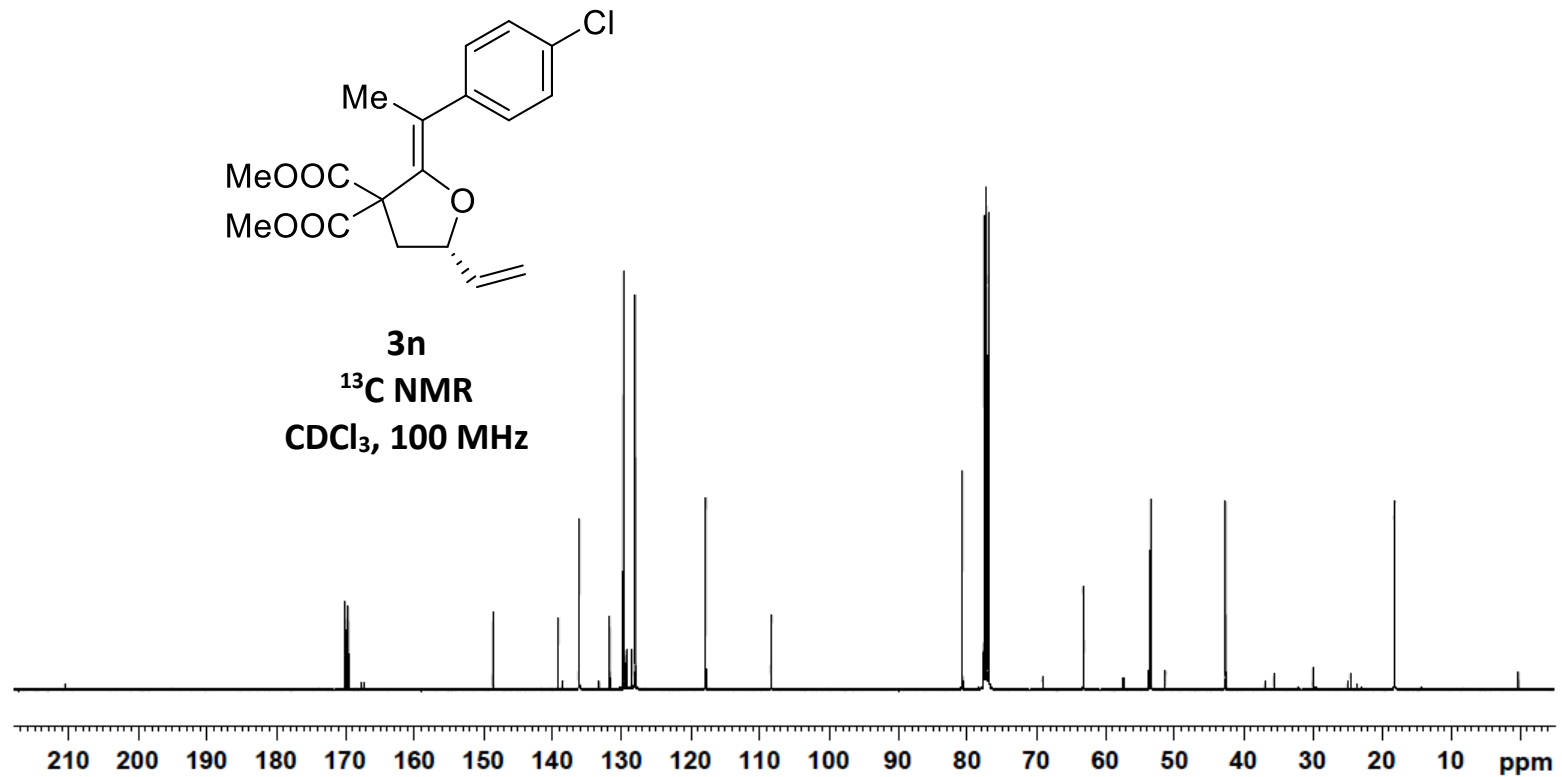



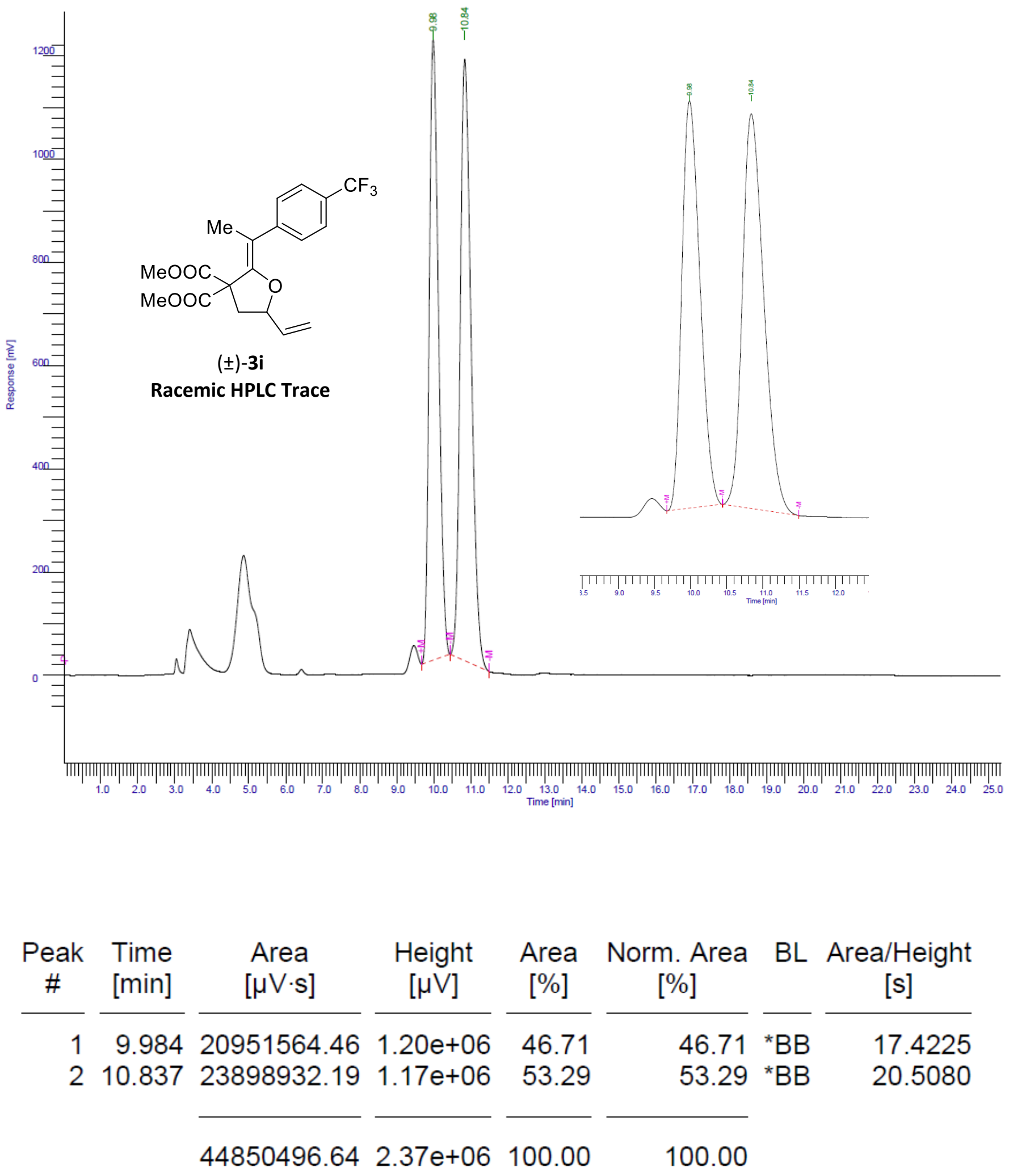


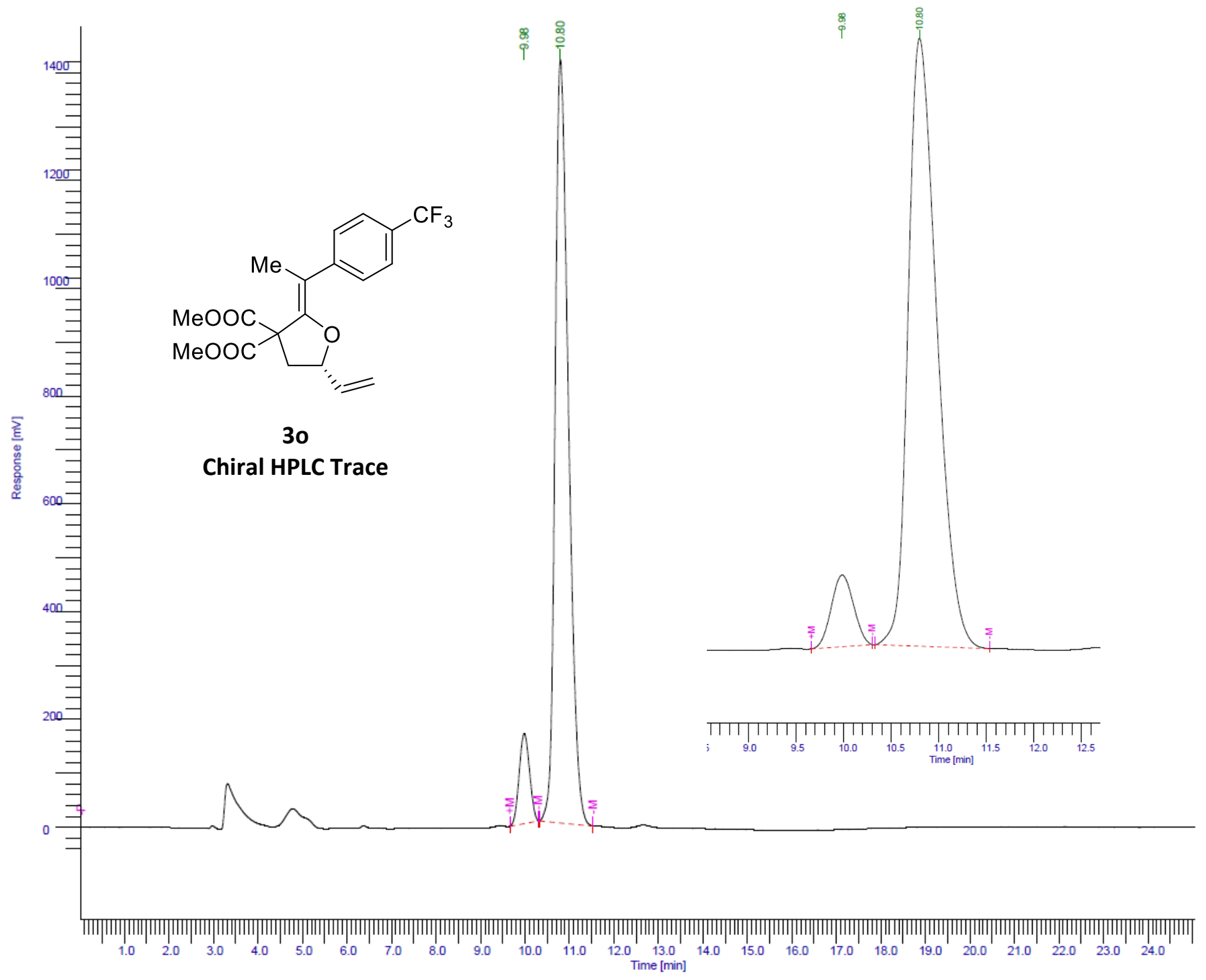

\begin{tabular}{|c|c|c|c|c|c|c|c|}
\hline $\begin{array}{c}\text { Peak } \\
\#\end{array}$ & $\begin{array}{l}\text { Time } \\
\text { [min] }\end{array}$ & $\begin{array}{l}\text { Area } \\
{[\mu \mathrm{V} \cdot \mathrm{s}]}\end{array}$ & $\begin{array}{l}\text { Height } \\
{[\mu \mathrm{V}]}\end{array}$ & $\begin{array}{c}\text { Area } \\
{[\%]}\end{array}$ & $\begin{array}{c}\text { Norm. Area } \\
{[\%]}\end{array}$ & $B L$ & $\begin{array}{c}\text { Area/Height } \\
\text { [s] }\end{array}$ \\
\hline & 9.983 & 2712619.46 & 167198.43 & 8.38 & 8.38 & ${ }^{*} \mathrm{BB}$ & 16.2240 \\
\hline & 10.796 & 29659580.13 & $1.42 e+06$ & 91.62 & 91.62 & ${ }^{*} \mathrm{BB}$ & 20.9292 \\
\hline & & 32372199.59 & $1.58 e+06$ & 100.00 & 100.00 & & \\
\hline
\end{tabular}




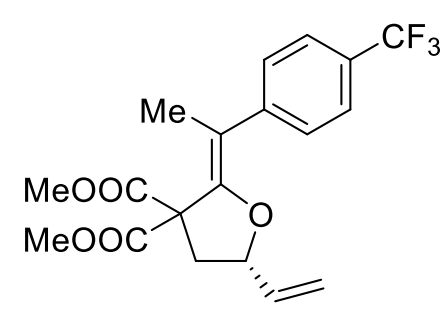

30

${ }^{1} \mathrm{H}$ NMR

$\mathrm{CDCl}_{3}, 400 \mathrm{MHz}$

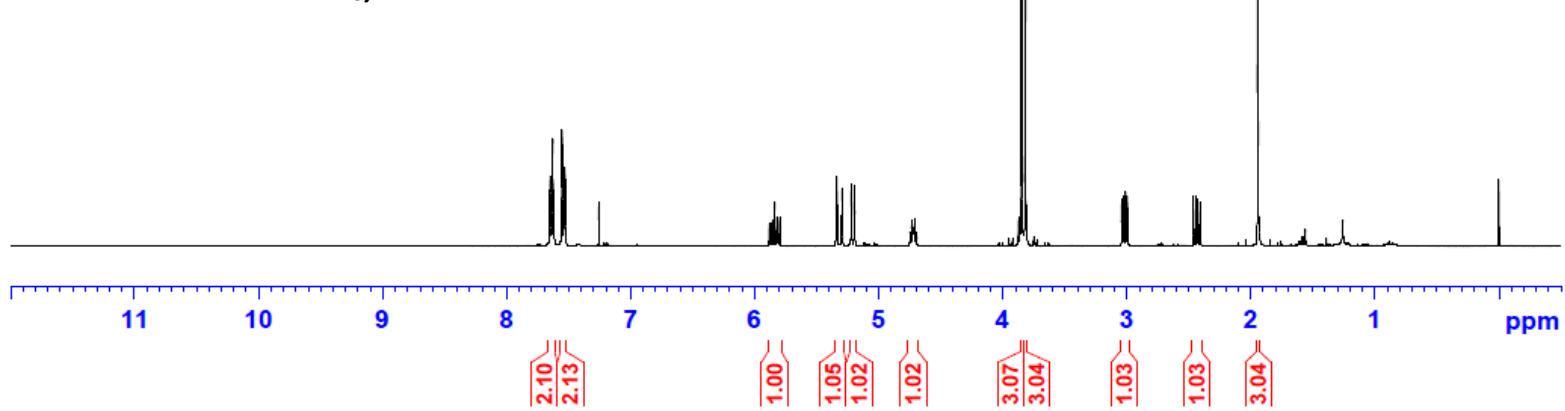

\%

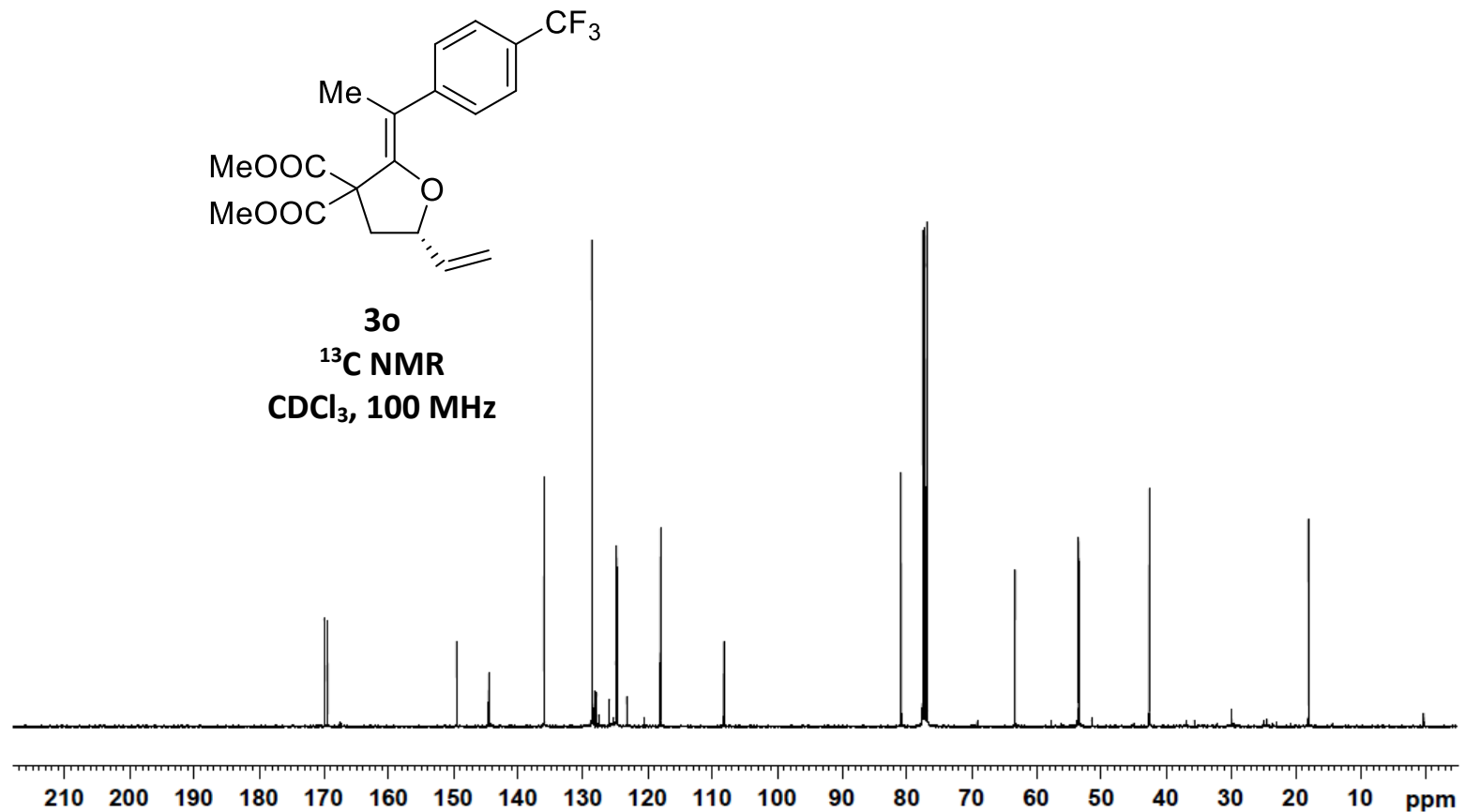



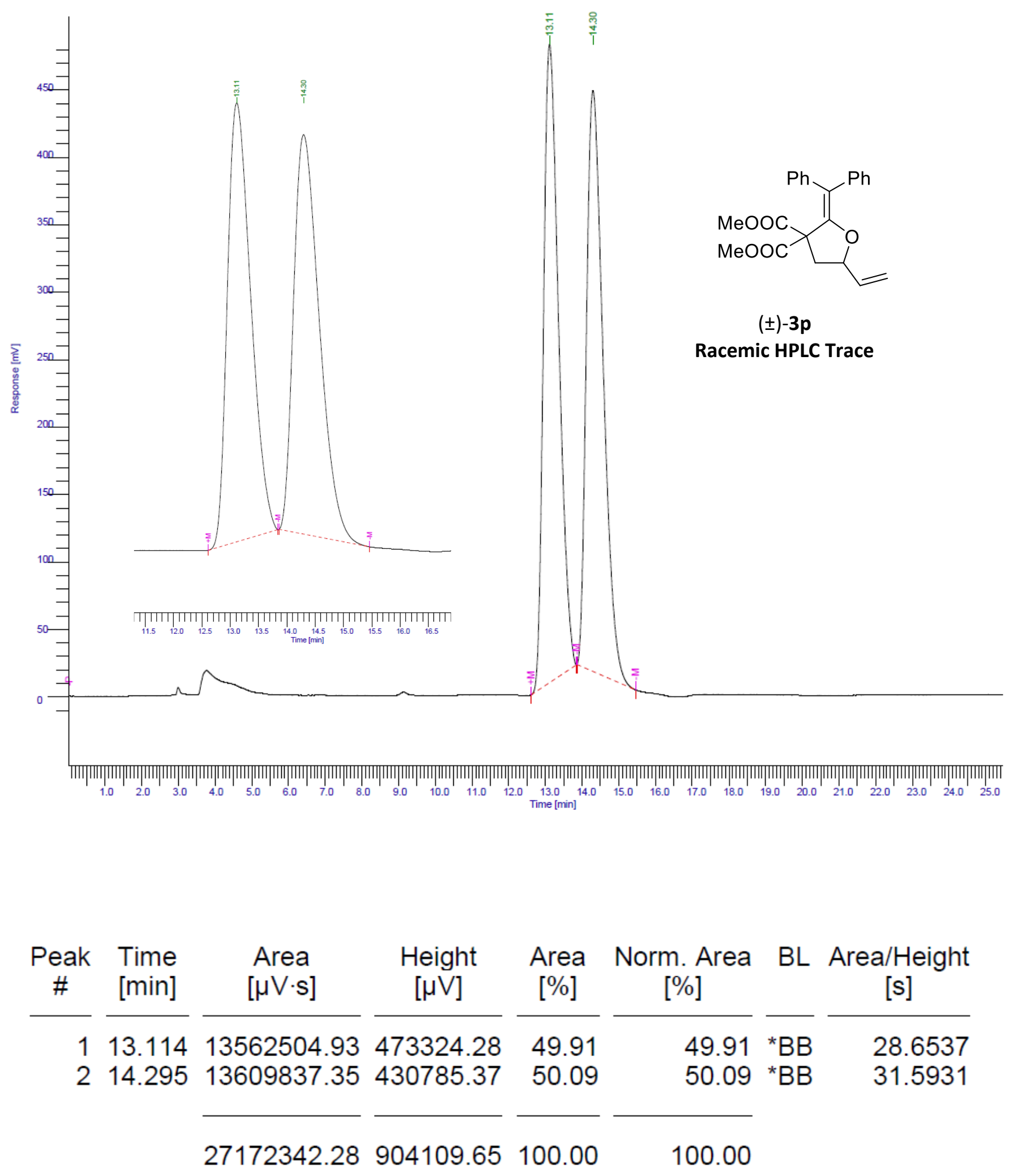


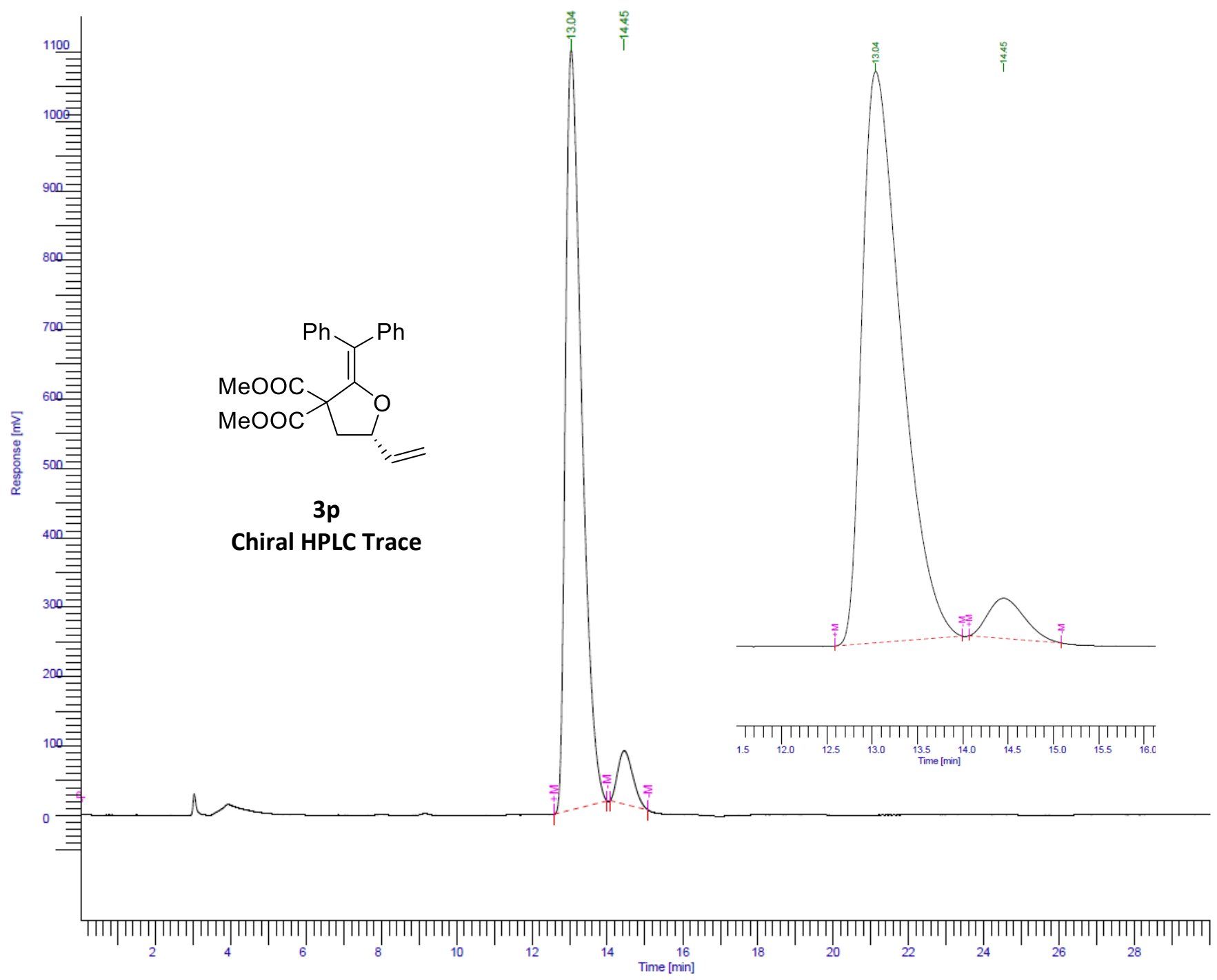

\begin{tabular}{|c|c|c|c|c|c|c|c|}
\hline $\begin{array}{l}\text { Peak } \\
\quad \#\end{array}$ & $\begin{array}{l}\text { Time } \\
\text { [min] }\end{array}$ & $\begin{array}{l}\text { Area } \\
{[\mu \mathrm{V} \cdot \mathrm{s}]}\end{array}$ & $\begin{array}{l}\text { Height } \\
{[\mu \mathrm{V}]}\end{array}$ & $\begin{array}{c}\text { Area } \\
{[\%]}\end{array}$ & $\begin{array}{c}\text { Norm. Area } \\
{[\%]}\end{array}$ & $B L$ & $\begin{array}{c}\text { Area/Height } \\
{[\mathrm{s}]}\end{array}$ \\
\hline & 13.039 & 32848901.31 & $1.10 e+06$ & 93.84 & 93.84 & *BB & 29.9844 \\
\hline & 14.449 & 2154456.99 & 76890.20 & 6.16 & 6.16 & ${ }^{*} \mathrm{BB}$ & 28.0199 \\
\hline & & 35003358.30 & $1.17 e+06$ & 100.00 & 100.00 & & \\
\hline
\end{tabular}




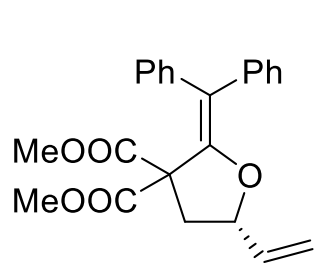

$3 p$

${ }^{1} \mathrm{H}$ NMR

$\mathrm{CDCl}_{3}, 400 \mathrm{MHz}$
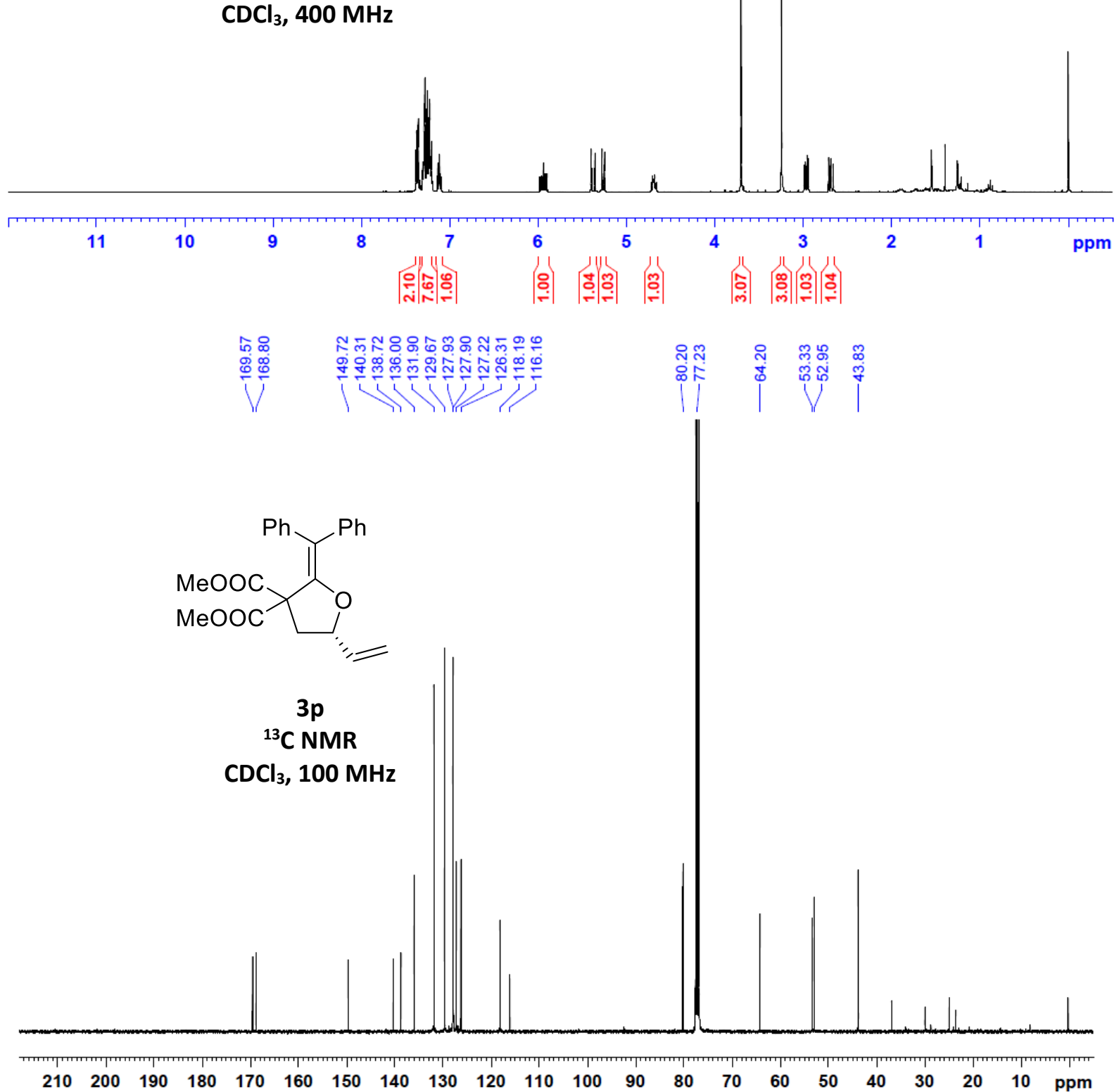


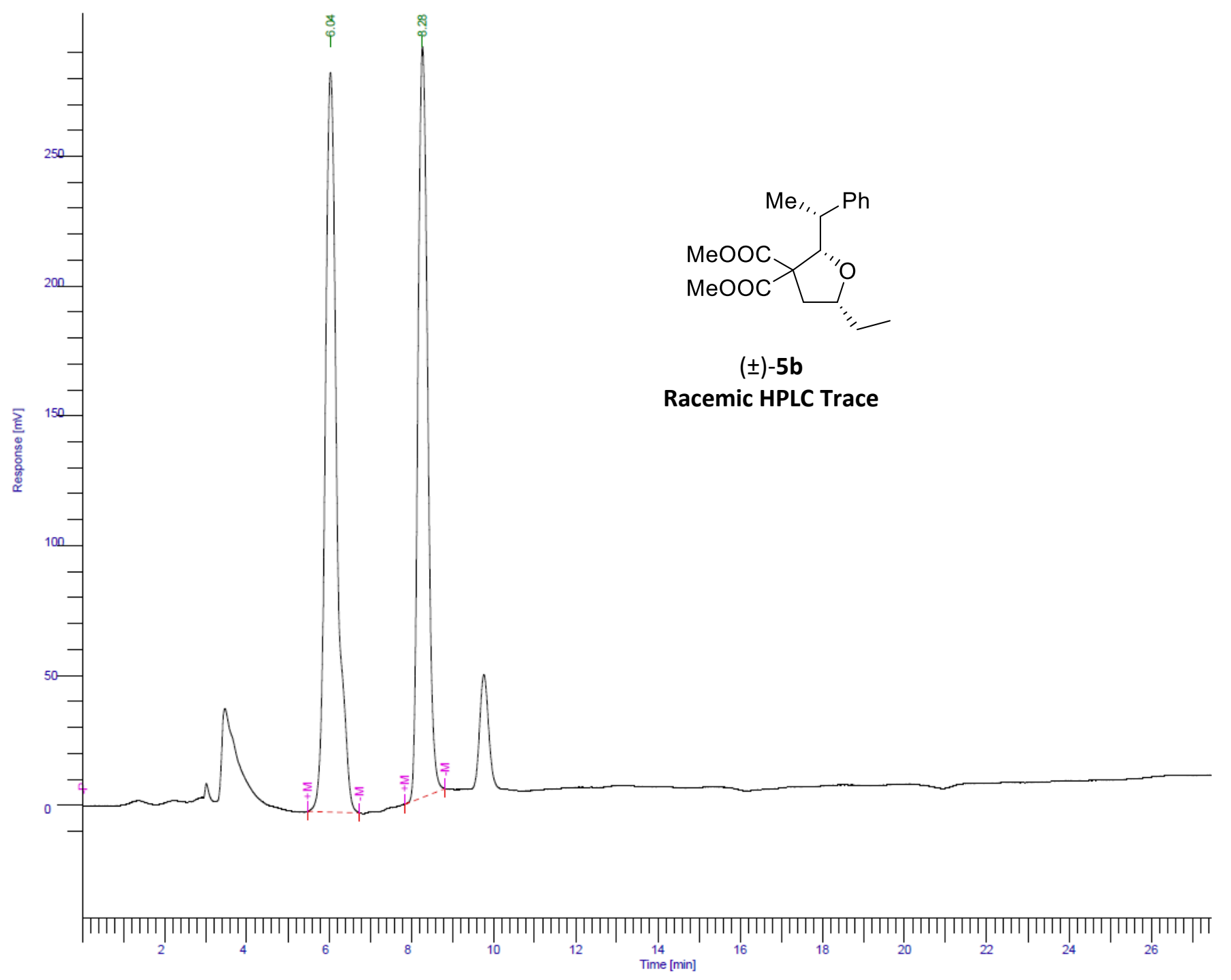

\begin{tabular}{|c|c|c|c|c|c|c|c|}
\hline $\begin{array}{c}\text { Peak } \\
\#\end{array}$ & $\begin{array}{l}\text { Time } \\
\text { [min] }\end{array}$ & $\begin{array}{l}\text { Area } \\
{[\mu \mathrm{V} \cdot \mathrm{s}]}\end{array}$ & $\begin{array}{l}\text { Height } \\
{[\mu \mathrm{V}]}\end{array}$ & $\begin{array}{c}\text { Area } \\
{[\%]}\end{array}$ & $\begin{array}{c}\text { Norm. Area } \\
{[\%]}\end{array}$ & $\mathrm{BL}$ & $\begin{array}{c}\text { Area/Height } \\
{[\mathrm{s}]}\end{array}$ \\
\hline & 6.037 & 5544547.66 & 284942.18 & 54.05 & 54.05 & \multirow{3}{*}{$\begin{array}{l}{ }^{*} \mathrm{BB} \\
{ }^{*} \mathrm{BB}\end{array}$} & \multirow{3}{*}{$\begin{array}{l}19.4585 \\
16.2960\end{array}$} \\
\hline & 8.275 & 4714467.08 & 289302.25 & 45.95 & 45.95 & & \\
\hline & & 10259014.74 & 574244.43 & 100.00 & 100.00 & & \\
\hline
\end{tabular}




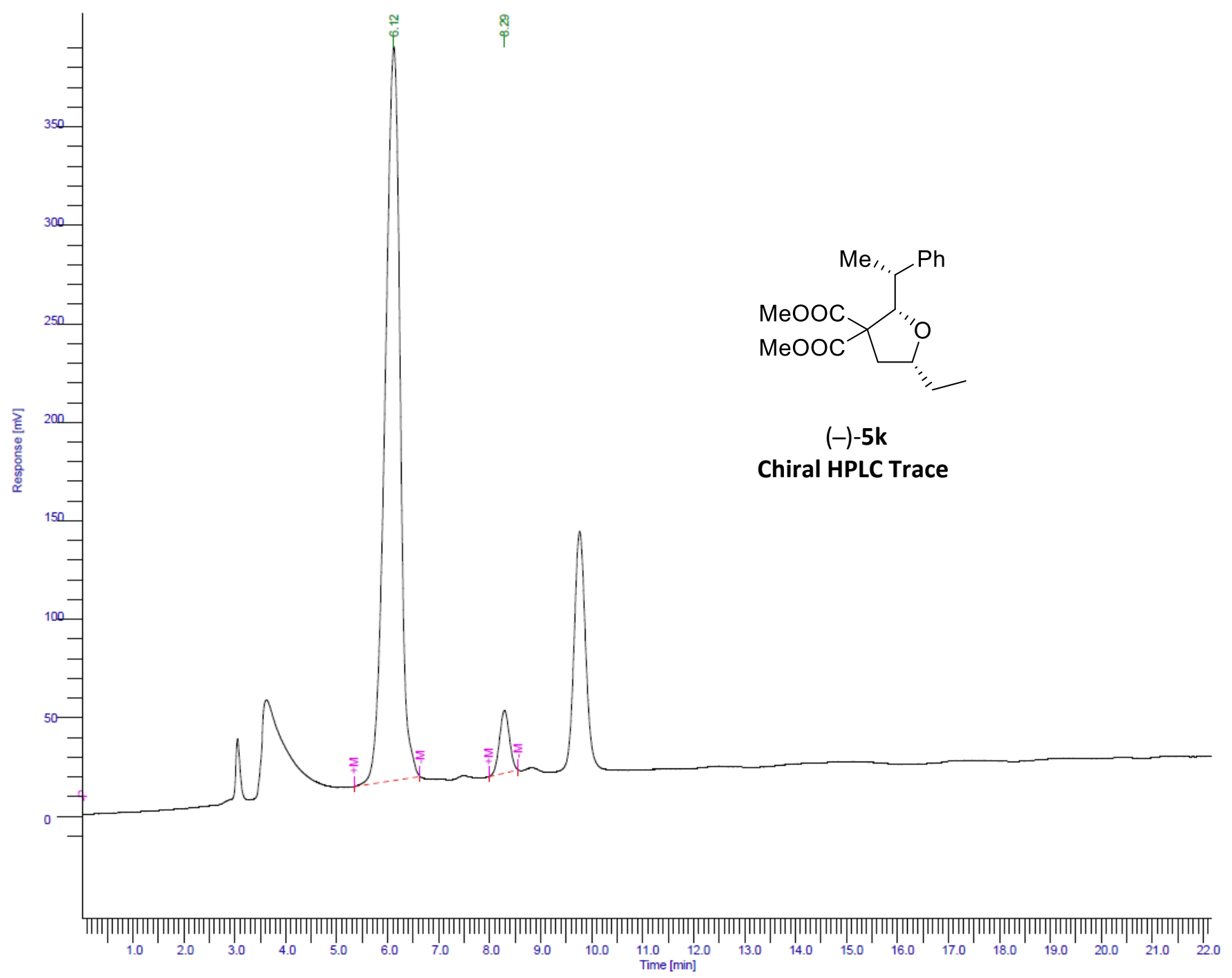

\begin{tabular}{|c|c|c|c|c|c|c|c|}
\hline $\begin{array}{l}\text { Peak } \\
\quad \#\end{array}$ & $\begin{array}{l}\text { Time } \\
\text { [min] }\end{array}$ & $\begin{array}{l}\text { Area } \\
{[\mu \mathrm{V} \cdot \mathrm{s}]}\end{array}$ & $\begin{array}{l}\text { Height } \\
{[\mu \mathrm{V}]}\end{array}$ & $\begin{array}{c}\text { Area } \\
{[\%]}\end{array}$ & $\begin{array}{c}\text { Norm. Area } \\
{[\%]}\end{array}$ & $B L$ & $\begin{array}{c}\text { Area/Height } \\
\text { [s] }\end{array}$ \\
\hline & 6.121 & 7619223.01 & 372765.88 & 94.65 & 94.65 & ${ }^{*} \mathrm{BB}$ & 20.4397 \\
\hline & 8.290 & 430428.49 & 31834.57 & 5.35 & 5.35 & ${ }^{*} \mathrm{BB}$ & 13.5208 \\
\hline & & 8049651.50 & 404600.46 & 100.00 & 100.00 & & \\
\hline
\end{tabular}




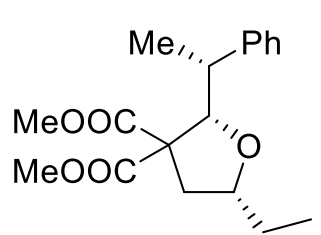

$( \pm)-5 b$

${ }^{1} \mathrm{H}$ NMR $\mathrm{CDCl}_{3}, 400 \mathrm{MHz}$

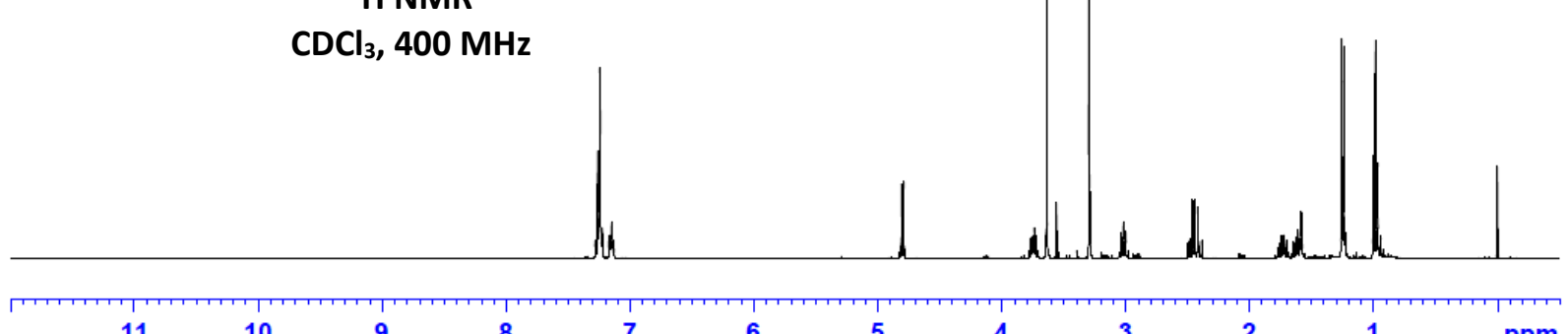

|

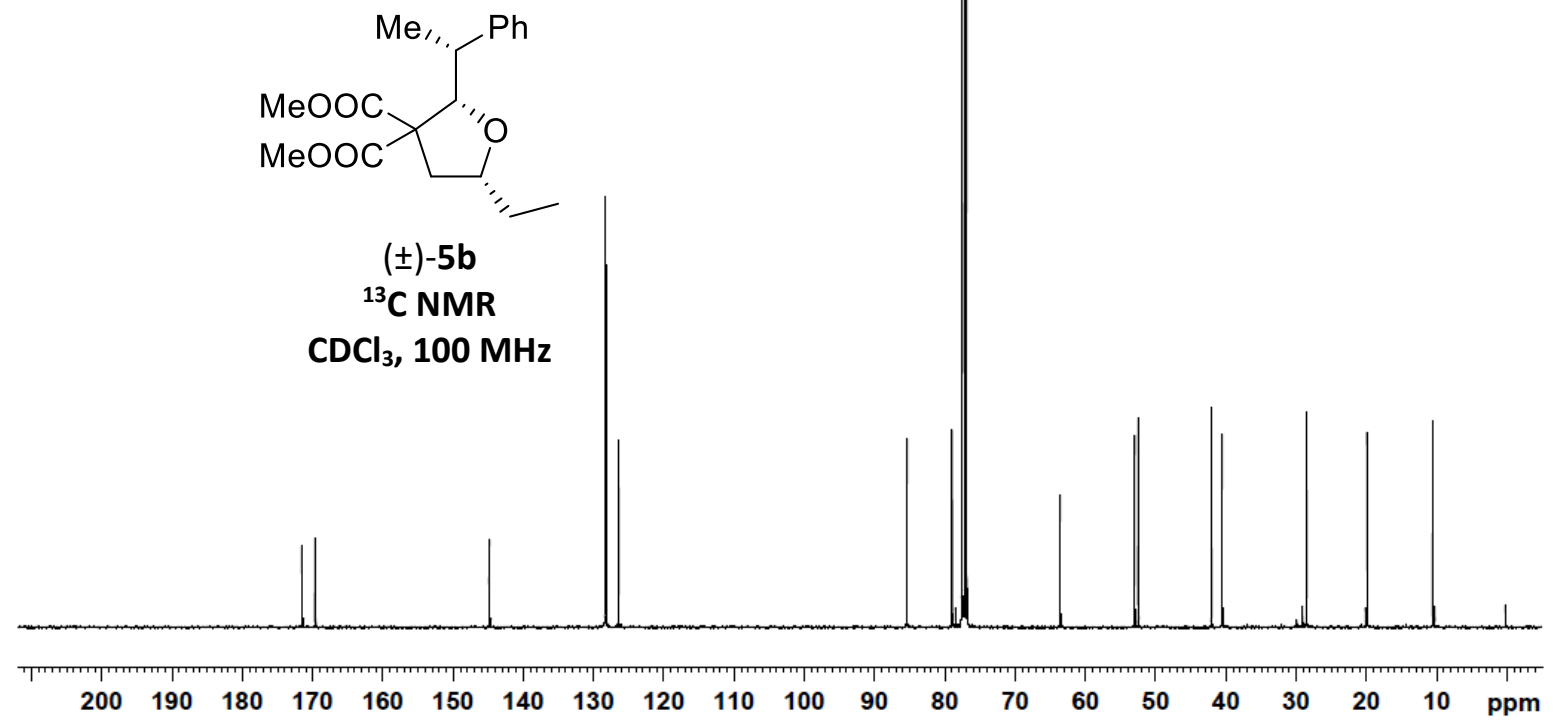




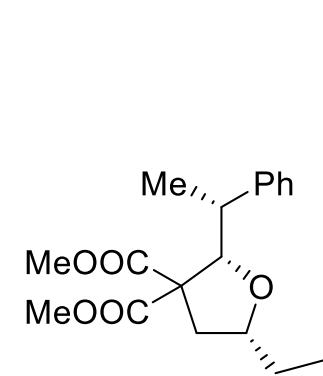

$(-)-5 k$

${ }^{1} \mathrm{H}$ NMR

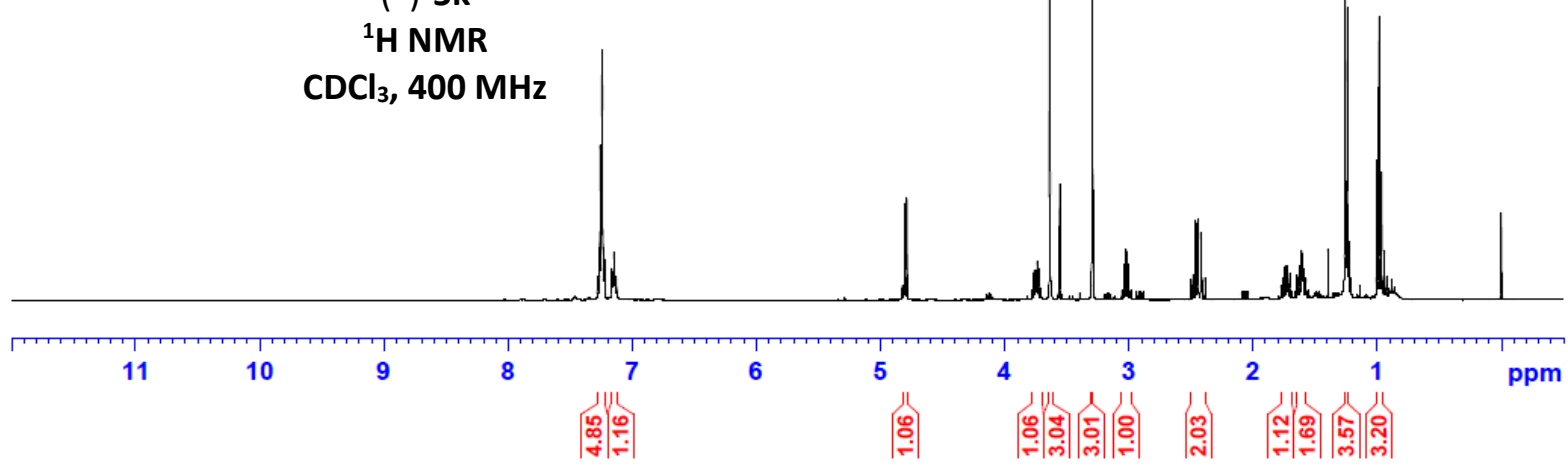

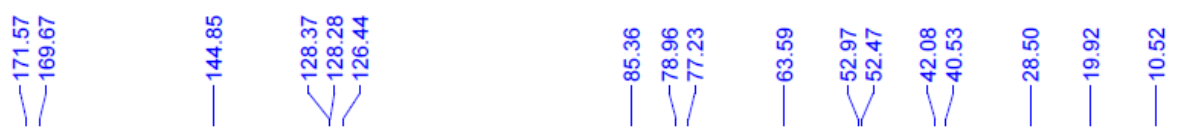

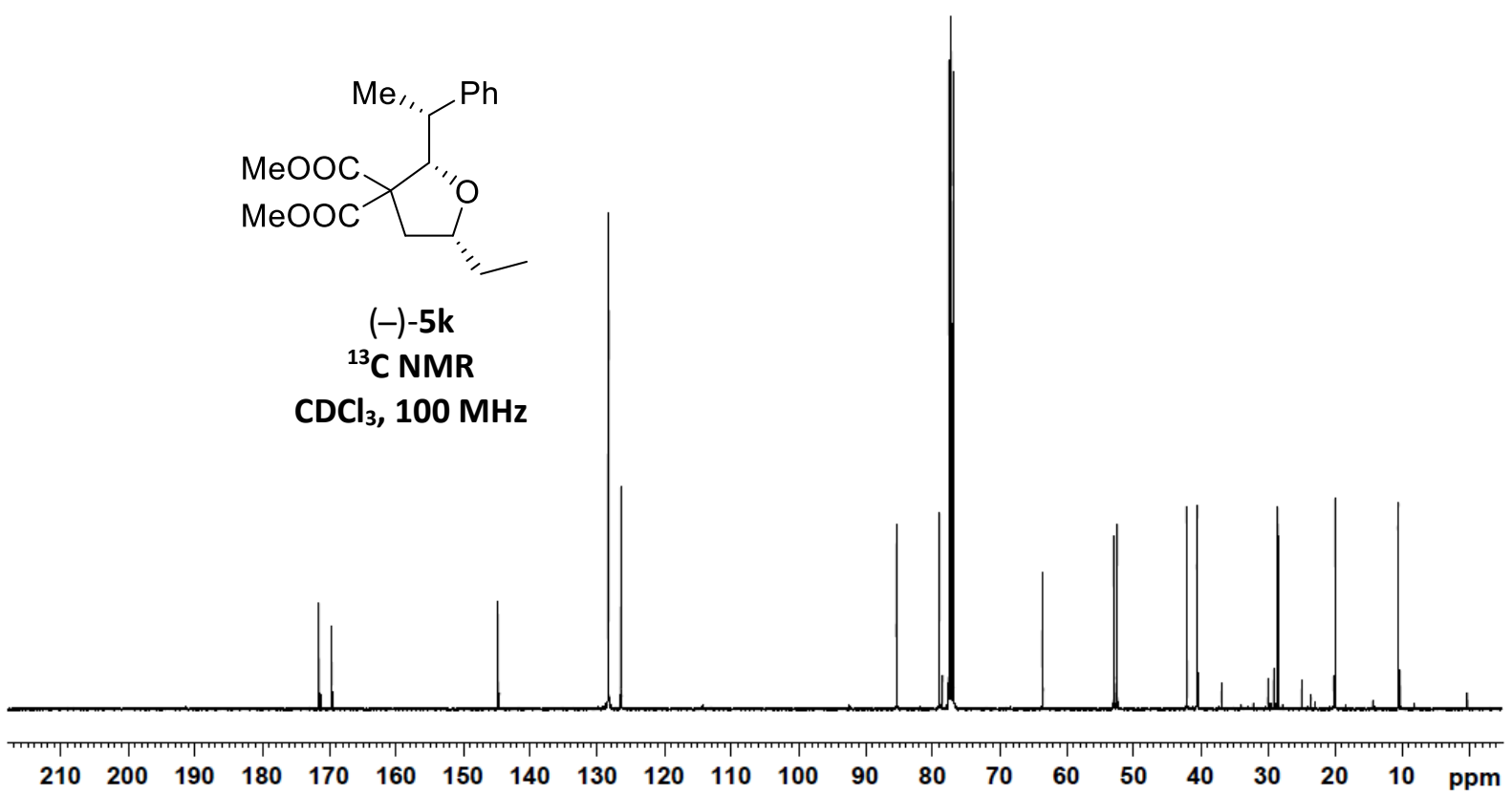


<smiles>C=C[C@H]1CC2(C(=O)NC(=O)NC2=O)/C(=C(\C)c2ccccc2)O1</smiles>

(+)-6k

${ }^{1} \mathrm{H}$ NMR

$\mathrm{C}_{3} \mathrm{D}_{6} \mathrm{O}, 400 \mathrm{MHz}$ |ำ<smiles>C1CCCCC1</smiles>

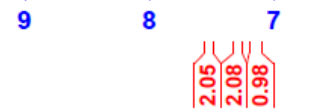

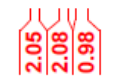
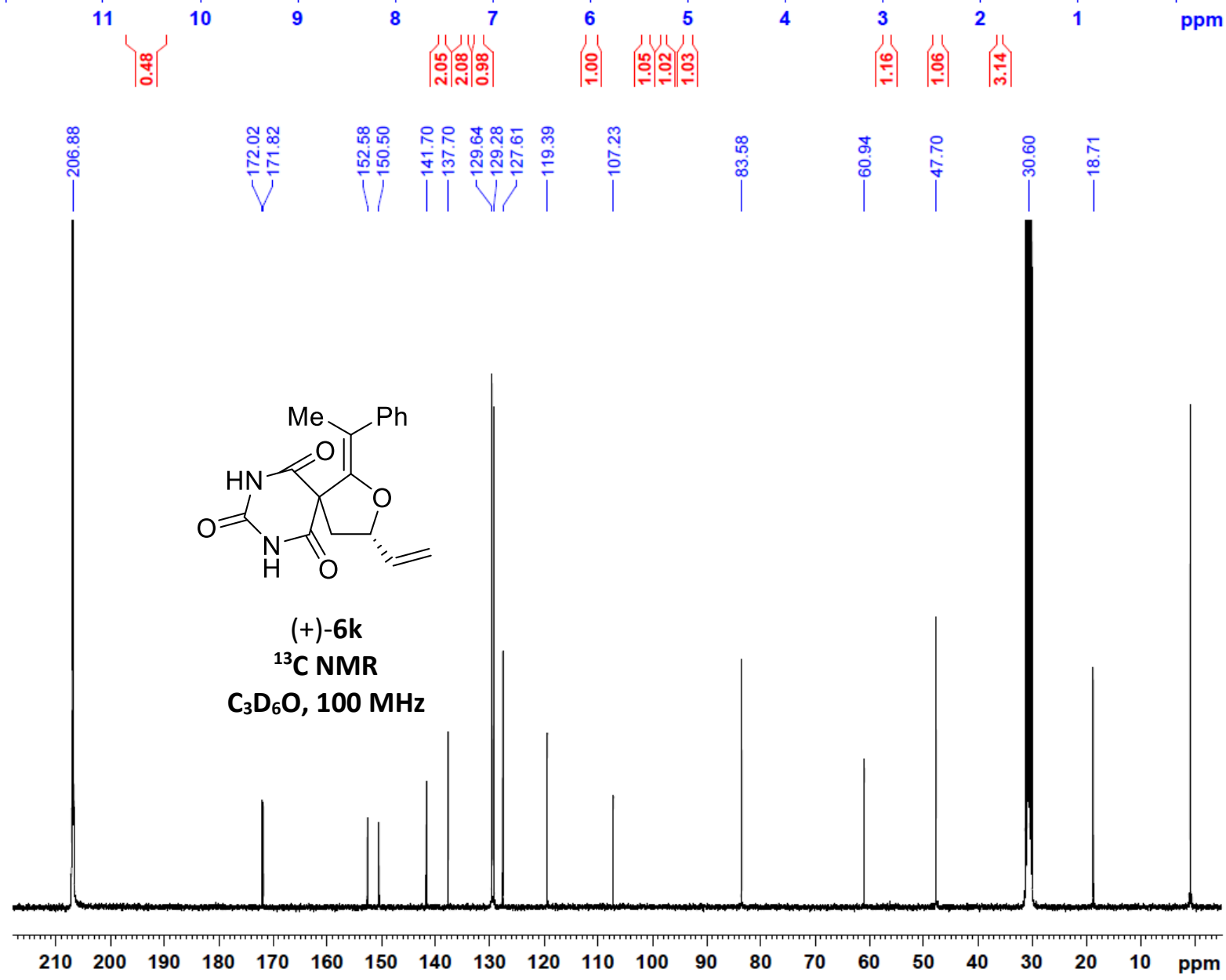


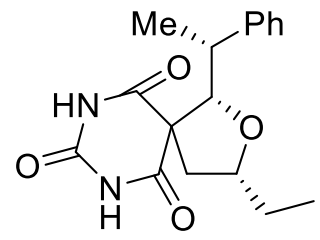

( \pm )-7b

${ }^{1} \mathrm{H}$ NMR $\mathrm{CDCl}_{3}, 400 \mathrm{MHz}$

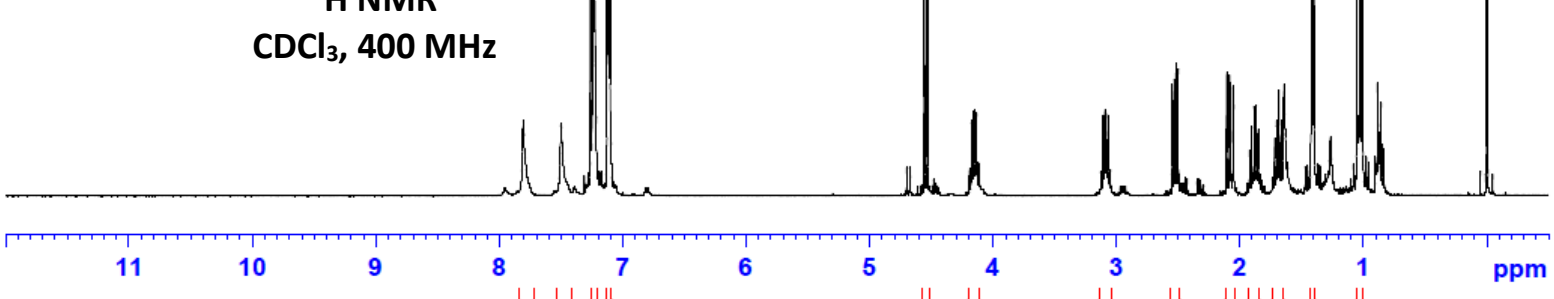

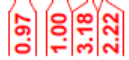

|ํ.

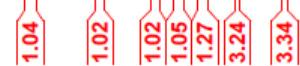

定足<smiles>CC[C@H]1C[C@]2(C(=O)NC(=O)NC2O)[C@H](C(C)c2ccccc2)O1</smiles>

( \pm )-7b

${ }^{13} \mathrm{C}$ NMR $\mathrm{CDCl}_{3}, 100 \mathrm{MHz}$

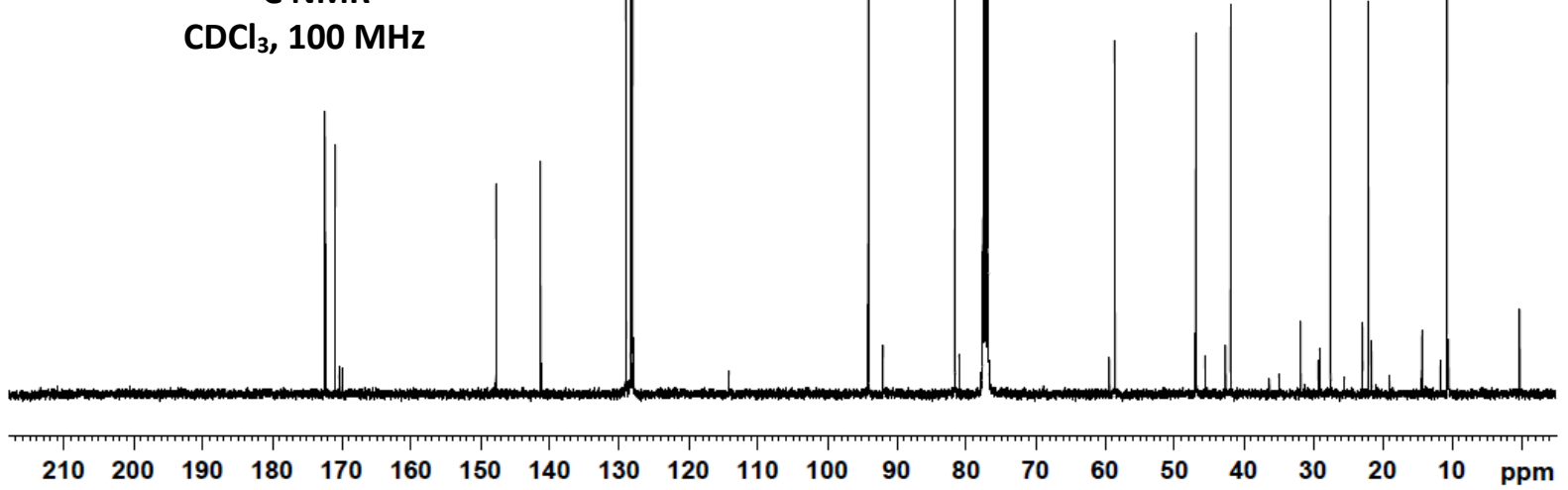




\section{X-ray crystal structure data}

Crystallographic data were collected at $100(1) \mathrm{K}$ on a Synergy, Dualflex, AtlasS2 diffractometer using $\mathrm{CuK \alpha}$ radiation $(\lambda=1.54184 \AA$ ). The structures were solved by dual space methods $\left(\mathrm{SHELXT}^{4}\right.$ ) and refined on $F^{2}$ using all the reflections (SHELXL-2018/35). Crystal data, data collection and structure refinement details are summarised Table S1.

Refs:

4. G.M. Sheldrick, Acta Cryst., 2015, A71, 3-8.

5. G.M. Sheldrick, Acta Cryst., 2015, C71, 3-8.

Table S1 Crystal Data and Structure Refinement

\begin{tabular}{|c|c|c|}
\hline Identification code & $(+)-6 k$ & $( \pm)-7 b$ \\
\hline Empirical formula & $\mathrm{C}_{17} \mathrm{H}_{16} \mathrm{~N}_{2} \mathrm{O}_{4}$ & $\mathrm{C}_{17} \mathrm{H}_{20} \mathrm{~N}_{2} \mathrm{O}_{4}$ \\
\hline Formula weight & 312.32 & 316.35 \\
\hline Temperature /K & $100.01(10)$ & $100.00(13)$ \\
\hline Crystal system & monoclinic & monoclinic \\
\hline Space group & $P 2_{1}$ & $12 / a$ \\
\hline$a / \AA ̊$ & $12.80150(10)$ & $10.5660(2)$ \\
\hline $\mathrm{b} / \AA ̊$ & $8.11190(10)$ & $17.6181(4)$ \\
\hline$c / \AA ̊$ & $15.13410(10)$ & $17.1644(4)$ \\
\hline$\beta /{ }^{\circ}$ & $108.0660(10)$ & $99.086(2)$ \\
\hline Volume $/ \AA^{3}$ & $1494.11(3)$ & 3155.10(12) \\
\hline z & 4 & 8 \\
\hline$\rho_{\text {calc }} \mathrm{g} / \mathrm{cm}^{3}$ & 1.388 & 1.332 \\
\hline$\mu / \mathrm{mm}^{-1}$ & 0.830 & 0.787 \\
\hline$F(000)$ & 656.0 & 1344.0 \\
\hline Crystal size $/ \mathrm{mm}^{3}$ & $0.145 \times 0.109 \times 0.049$ & $0.211 \times 0.055 \times 0.044$ \\
\hline $2 \theta$ range for data collection $/{ }^{\circ}$ & 6.142 to 149.096 & 7.238 to 133.18 \\
\hline Reflections collected & 34812 & 14222 \\
\hline Independent reflections, $R_{\text {int }}, R_{\text {sigma }}$ & $6005,0.0234,0.0149$ & $2799,0.0137,0.0087$ \\
\hline Data/restraints/parameters & $6005 / 1 / 429$ & $2799 / 1184 / 330$ \\
\hline Goodness-of-fit on $\mathrm{F}^{2}$ & 1.037 & 1.052 \\
\hline Final R indexes $[1>=2 \sigma(I)]$ & $R_{1}=0.0287, w R_{2}=0.0745$ & $R_{1}=0.0579, w R_{2}=0.1640$ \\
\hline Final R indexes [all data] & $R_{1}=0.0298, w R_{2}=0.0754$ & $R_{1}=0.0615, w R_{2}=0.1679$ \\
\hline Largest diff. peak/hole / e $\AA^{-3}$ & $0.29 /-0.19$ & $0.32 /-0.24$ \\
\hline Flack parameter & $0.00(5)$ & n/a \\
\hline CCDC number & 1895670 & 1895669 \\
\hline
\end{tabular}




\section{Experimental}

The data were collected at 100(1)K on a Synergy, Dualflex, AtlasS2 diffractometer using $\mathrm{Cu} K_{\alpha}$ radiation $\left(\lambda=1.54184 \AA\right.$ ) and the CrysAlis PRO 1.171.40.29a suite ${ }^{6}$. Using SHELXLE ${ }^{7}$ and Olex $2^{8}$ the structure was solved by dual space methods $\left(\mathrm{SHELXT}^{9}\right.$ ) and refined on $F^{2}$ using all the reflections (SHELXL$2018 / 3^{10}$ ). All the non-hydrogen atoms were refined using anisotropic atomic displacement parameters. Hydrogen atoms were inserted at calculated positions using a riding model, except for the hydrogens bonded to nitrogen in (+)-6k, which were located from difference maps and their coordinates refined. Absolute configuration was unambiguously determined for the chiral structure, (+)-6k. Crystal data, data collection and structure refinement details are summarised in Table S1.

6. Rigaku Oxford Diffraction, (2018), CrysAlisPro Software system, version 1.171.39.27b, Rigaku Corporation, Oxford, UK.

7. C.B. Hübschle, G.M. Sheldrick and B. Dittrich. J. Appl. Cryst., 2011, 44, 12811284.

8. O.V. Dolomanov, L.J. Bourhis, R.J. Gildea, J.A.K. Howard \& H. Puschmann. J. Appl. Cryst., 2009, 42, 339-341

9. G.M. Sheldrick, Acta Cryst., 2015, A71, 3-8.

10. G.M. Sheldrick, Acta Cryst., 2015, C71, 3-8.

$(+)-6 \mathrm{~K} \mathrm{C}_{17} \mathrm{H}_{16} \mathrm{~N}_{2} \mathrm{O}_{4}$

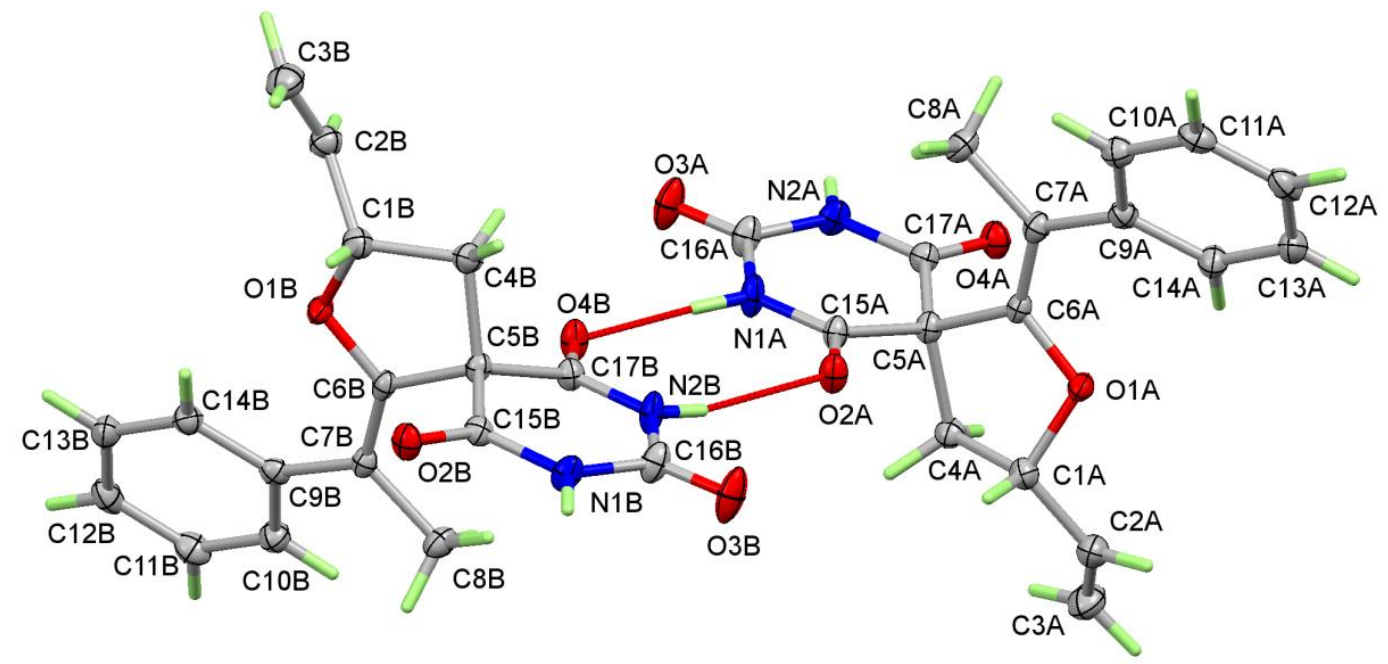

Fig $51.50 \%$ ellipsoid plot showing labelling scheme

Slow crystallization of crude $\mathbf{6 k}$ from a mixture of hexanes and dichloromethane afforded pure (+)-6k as a colorless crystalline solid. The compound crystallised in the chiral space group $P 2_{1}$, with two independent molecules in the asymmetric unit. Both molecules have the same chirality but somewhat different conformations, particularly the phenyl and alkene group (for example the torsion angles: C6A- 
$\mathrm{C} 7 \mathrm{~A}-\mathrm{C} 9 \mathrm{~A}-\mathrm{C} 10 \mathrm{~A},-153.66(19)^{\circ}$ and $\left.\mathrm{C} 6 \mathrm{~B}-\mathrm{C} 7 \mathrm{~B}-\mathrm{C} 9 \mathrm{~B}-\mathrm{C} 10 \mathrm{~B}, 160.06(19)^{\circ}.\right)$

The absolute configuration is unambiguously determined from the crystallographic data (Flack parameter $0.00(5))$.

Each "A" molecule makes two H-bonds with an adjacent "B" molecule and two other H-bonds to different neighbours. The hydrogen bonding links the molecules into zig-zag layers perpendicular to the $c$ axis.

Hydrogen-bond geometry $(\AA ⿻$, o for (+)-6k

\begin{tabular}{|l|l|l|l|l|}
\hline$D-\mathrm{H} \cdots A$ & $D-\mathrm{H}$ & $\mathrm{H} \cdots A$ & $D \cdots A$ & $D-\mathrm{H} \cdots A$ \\
\hline $\mathrm{N} 1 A-\mathrm{H} 1 A A \cdots \mathrm{O} 4 B$ & $0.91(3)$ & $1.96(3)$ & $2.868(2)$ & $177(2)$ \\
\hline $\mathrm{N} 2 A-\mathrm{H} 2 A A \cdots \mathrm{O} 4 B^{\mathrm{i}}$ & $0.81(3)$ & $2.25(3)$ & $3.017(2)$ & $159(2)$ \\
\hline $\mathrm{N} 1 B-\mathrm{H} 1 B \cdots \mathrm{O} 2 A^{\mathrm{ii}}$ & $0.87(3)$ & $2.16(3)$ & $2.990(2)$ & $158(2)$ \\
\hline $\mathrm{N} 2 B-\mathrm{H} 2 B A \cdots \mathrm{O} 2 A$ & $0.79(3)$ & $2.15(3)$ & $2.931(2)$ & $169(3)$ \\
\hline
\end{tabular}

Symmetry codes: (i) $-x+1, y+1 / 2,-z+1$; (ii) $-x, y-1 / 2,-z+1$.
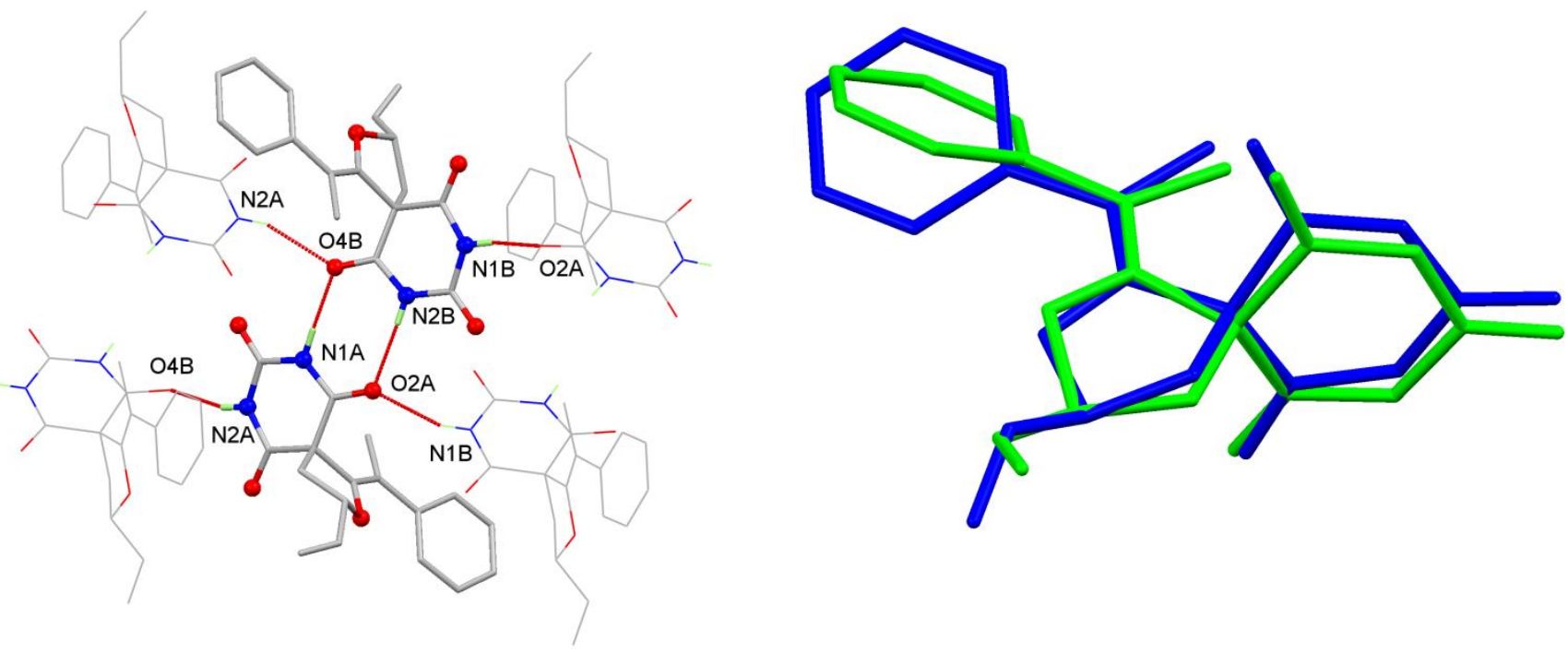

Fig S2. Left: H-bonding to neighbouring molecules. Right: superposition of molecules A (green) and B (blue). 


\section{$( \pm)-7 \mathrm{~b} \mathrm{C} \mathrm{C}_{17} \mathrm{H}_{20} \mathrm{~N}_{2} \mathrm{O}_{4}$}
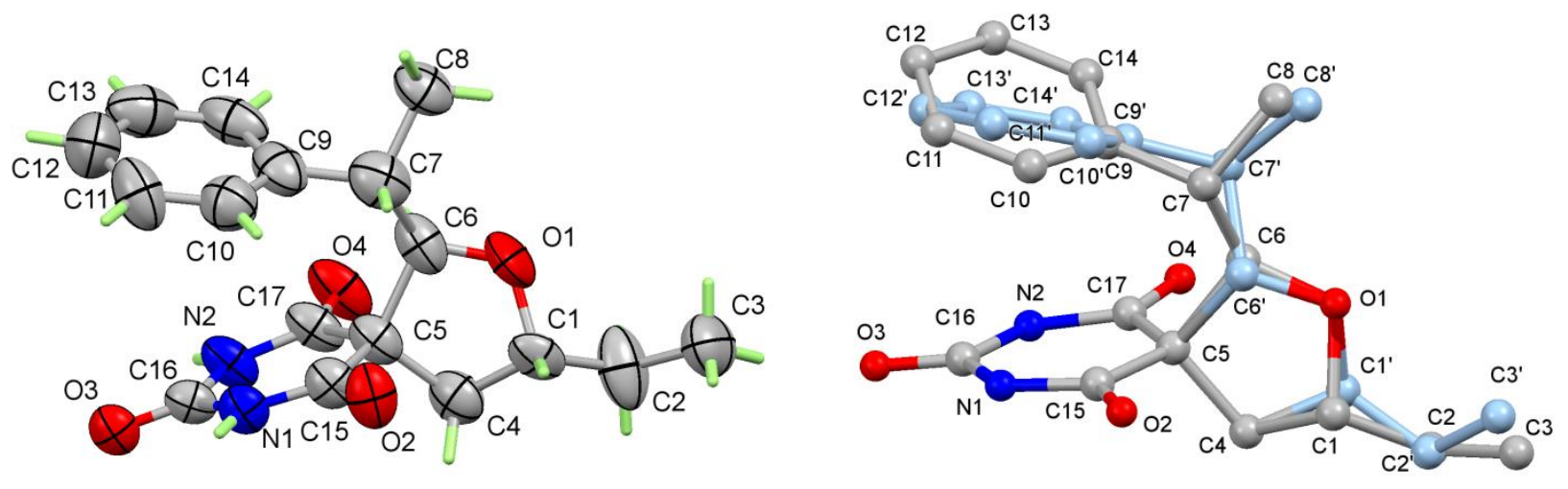

Fig S3. Left: 50\% ellipsoids, second component omitted. Right: superimposed components.

Slow crystallization of crude $( \pm)-7 \mathbf{b}$ from a mixture of hexanes and acetone afforded pure $( \pm)-7 \mathbf{b}$ as a colorless crystalline solid. The structure was refined in 12/a, where the asymmetric unit contains $50 \%$ of each enantiomer superimposed so that the triazine rings coincide. This disorder remained apparent when the symmetry was reduced (P2, Pc, 12 or la), so the simplest model was chosen.

The molecules are linked into $\mathrm{H}$-bonded chains running parallel to the $a$ axis and involving the non-disordered sections of the molecule.

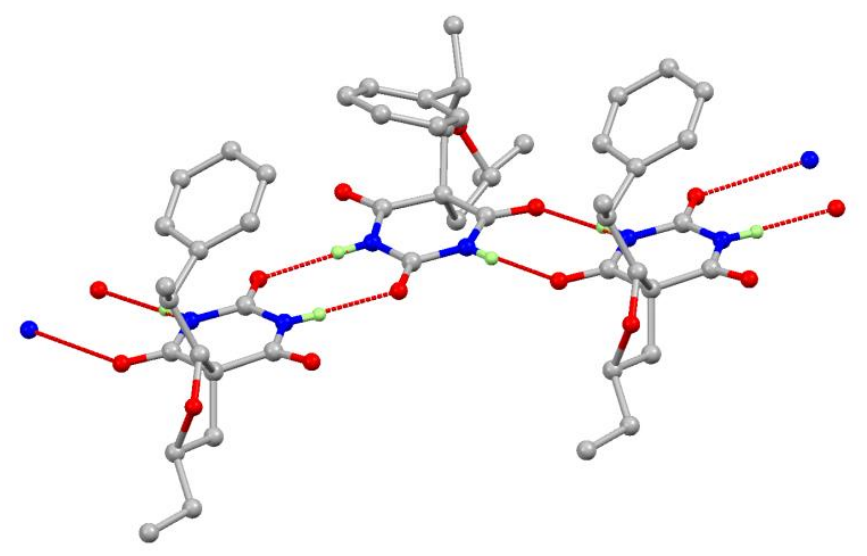

Fig S4. $H$ bonding in $( \pm)-7 \mathbf{b}$

Hydrogen-bond geometry $(\AA ̊ \stackrel{\circ}{)})$ for $( \pm)-7 \mathbf{b}$

\begin{tabular}{|l|l|l|l|l|}
\hline$D-\mathrm{H} \cdots A$ & $D-\mathrm{H}$ & $\mathrm{H} \cdots A$ & $D \cdots A$ & $D-\mathrm{H} \cdots A$ \\
\hline $\mathrm{N} 1-\mathrm{H} 1 \cdots \mathrm{O} 2^{\mathrm{i}}$ & 0.86 & 2.04 & $2.893(2)$ & 172 \\
\hline $\mathrm{N} 2-\mathrm{H} 2 \cdots 3^{\mathrm{ii}}$ & 0.86 & 2.04 & $2.898(2)$ & 175 \\
\hline $\mathrm{N} 1^{\prime}-\mathrm{H}^{\prime} \cdots \mathrm{O}^{\prime i}$ & 0.86 & 2.04 & $2.893(2)$ & 172 \\
\hline $\mathrm{N} 2^{\prime}-\mathrm{H}^{\prime} \cdots 3^{\prime i i}$ & 0.86 & 2.04 & $2.898(2)$ & 175 \\
\hline
\end{tabular}


Symmetry codes: (i) $-x+1 / 2, y,-z+1$; (ii) $-x+3 / 2, y,-z+1$; (iii) $x-1 / 2,-y+1, z$. 